\title{
DERIVATION OF A BGK MODEL FOR REACTING GAS MIXTURES*
}

\author{
STÉPHANE BRULL ${ }^{\dagger}$ AND JACQUES SCHNEIDER ${ }^{\ddagger}$
}

\begin{abstract}
In this paper we derive a new relaxation model for reacting gas mixtures. We prove that this model satisfies the fundamental properties (equilibrium states, conservation laws, $\mathrm{H}$-theorem, ...). We also consider the slow reaction regime. In this case a rigorous Chapman-Enskog procedure is performed and Navier-Stokes equations are derived.
\end{abstract}

Key words. Kinetic theory, gas mixtures, BGK models, entropy minimization, hydrodynamic limit.

AMS subject classifications. 35Q20, 35Q35.

\section{Introduction}

The modeling of reacting gas mixtures is essentially based on the microscopic phenomena that occur when particles are colliding. Part of those collisions are non reactive while the others lead to different species and feature the chemical reactions. The same distinction is done at the kinetic level by adding to the classical collision operators for gas mixtures collision operators that only feature chemical reactions. A quite general setting of those equations can be found in [23. It is however interesting to focus on the case where only one chemical reaction is considered. A simplified model has been proposed by Rossani and Spiga [32] where each species has only one degree of internal energy and where elastic collisions occur for the non reactive part of the collision operators. A generalization of this model to different degrees of internal energies was proposed in 25]. In particular they have considered a four species gas mixture with a reversible chemical reaction

$$
A^{1}+A^{2} \leftrightarrow A^{3}+A^{4}
$$

(Here $A^{i}$ stands for the species $i$ ).

The main motivation of this paper is the derivation of a relaxation model based on the simplified model of Rossani and Spiga. Our concerns are to include as many physical properties as possible (positivity, equilibrium states, conservation laws, $\mathrm{H}$ theorem) as well as minimizing computational cost. Such a model is the well known BGK model [4 for monoatomic gas. But its drawback is that it cannot lead to the proper Prandtl number at the Navier-Stokes level. This problem has been overcome by Holway [28] who introduced the so-called ES-BGK model. When inert gas mixtures are considered, cross effects such as "mass" diffusion (Fick law) or thermo diffusion (Soret law) occur. The derivation of BGK models giving the right transport coefficients at the hydrodynamic limit is much more complicated. For example Kosuge 29] has derived a BGK operator that is able to approximate those coefficients but which guarantees neither the non negativity of the distribution functions nor the entropy decay. Andries et al. have successfully addressed the later problems [1] but their model is valid only for Maxwellian molecules and thus does not respond to the above requirements. The authors of the present paper have introduced a new concept of relaxation

\footnotetext{
${ }^{*}$ Received: February 4, 2013; accepted (in revised form): August 31, 2013. Communicated by Francois Golse.

†Univ. Bordeaux, IMB, UMR 5251, F-33400 Talence, France (Stephane.Brull@math.ubordeaux1.fr).

${ }^{\ddagger}$ University of Toulon, IMATH, EA 2134, 83957 La Garde, France (jacques.schneider@univ-tln.fr).
} 
coefficients on non-conserved moments which allows one to construct BGK type models. A first achievement was to recover the ES-BGK model in the monoatomic and polyatomic cases [12, 13]. But this idea extends to more general cases and we have derived in [14] a relaxation model allowing one to recover the correct Fick law at the hydrodynamic and enjoying the required mathematical (and physical) properties.

Coming back to reactive gas mixtures, many BGK models were constructed by Groppi, Spiga and co-authors (see for example [9] for a complete bibliography) basing upon the "simplified" Boltzmann equation of Rossani and Spiga [32. Those models always feature only one relaxation operator per species that includes both mechanical and chemical reactions. For the whole set of models the mechanical part is based on [1] where Maxwellian molecules are considered. Hence the hydrodynamic limits in the case of slow reactions lead to the Navier-Stokes equations with incorrect transport coefficients. The chemical contributions depend on the approximation of the chemical collision operators. There are essentially two types of models. The first ones are constructed using estimations of the exchanges of mass, momentum, and (kinetic plus internal energy) per species ([26] and [9]). So in some sense those models mimic the one by Andries et al. [1]. However those values cannot be computed in general even under the assumption of Maxwellian molecules and strong restrictions must be made - distribution functions at mechanical equilibrium (Maxwellian distributions) to overcome this problem. This means that those models are essentially adapted to the case of slow reactions where the chemical reactions occur when the distribution of molecules are closed to mechanical equilibrium. The second model may be called "the" BGK model for mechanical plus chemical reactions 24. The macroscopic parameters of the Maxwellian attractors are given under the requirements of number atoms conservation and conservation laws and bound together with some type of mass action law. This model enjoys the classical H-theorem for chemically reacting gas mixtures, unlike to the first class of models for which it is very unlikely that this property holds.

We intend in this paper to take advantage on one side on the modeling for inert gas mixtures and on the other side on the modeling for chemical reactions. Our methodology is based on a splitting between a mechanical operator and a chemical one as for the full Boltzmann equation. This procedure is particularly adapted to the case of slow reaction regime. More precisely we simply add to the model derived in 14] a chemical relaxation operator directly derived from [24. However we remark that while the chemical BGK operator seems to be more adapted to the case of fast chemical reactions, our choice relies upon the possibility to prove an $\mathrm{H}$-theorem. In doing so the present paper must be considered more as an example of the splitting methodology than an attempt to match the best approximation of the chemical contribution in the case of slow reaction regime.

The paper is organized as follows. In Section 3 we make a short review of different Boltzmann equations for reacting mixtures. In the next section we show that the construction of the mechanical model can be achieved either by using experimental values (Fick law) or by algorithms developed by Ern and Giovangigli [19]. Special attention is given as to the definition of the relaxation coefficients for the chemical model. Then we show that the whole relaxation model satisfies the H-theorem and that equilibrium states are Maxwellian functions which densities and temperature are bound together with the mass action law. Finally Section 5 is devoted to the derivation of the Euler and the Navier-Stokes system in the slow reacting regime. The ChapmanEnskog procedure yields a first formulation of the Navier-Stokes. We focus on the 
calculation of the chemical terms at the first order. The second formulation of those equations (Section 5.3.2) is obtained by using the work of Kurochkin, Makarenko, and Tirskii [30] on the transport coefficients and the Onsager relations. Thus the NavierStokes equations are recast in the classical framework of polyatomic gases (see for example [23). This makes possible the comparison between the different coefficients (species multicomponent diffusion, thermal diffusion, etc.) that are obtained on one side with our model and on the other side with the usual theory. This also allows one to entirely determine the definition of our model as already mentioned above.

\section{Notations}

Consider a gas mixture with $p$ components. $f_{i}(t, x, v)$ (or for short $f_{i}, i \in[1, p]$ with $\left.\mathbf{f}:=\left(f_{1}, \cdots, f_{p}\right)\right)$ represents the distribution function of a given species $i$.

We denote with $n^{i}, \rho^{i}, u^{i}, \mathcal{E}^{i}$, and $T^{i}$ the macroscopic quantities representing respectively the density and mass per unit volume, average velocity, internal energy per unit volume, and finally temperature of a given species $i$. They are defined by the following relations:

$$
\begin{array}{r}
n^{i}=\int_{\mathbb{R}^{3}} f_{i} d \mathbf{v}, \quad \rho^{i}=m_{i} n^{i}, \quad n^{i} \mathbf{u}^{i}=\int_{\mathbb{R}^{3}} \mathbf{v} f_{i} d \mathbf{v}, \\
\mathcal{E}^{i}=n^{i}\left(\frac{3}{2} k_{B} T^{i}+E_{i}\right)=\int_{\mathbb{R}^{3}}\left(\frac{m_{i}}{2}\left\|\mathbf{v}-\mathbf{u}^{i}\right\|^{2}+E_{i}\right) f_{i} d \mathbf{v},
\end{array}
$$

where $k_{B}$ is the Boltzmann constant and \|\| is the Euclidean norm in $\mathbb{R}^{3} . E_{i}$ represents the internal energy of species $i$ due to chemical links. In the same way macroscopic quantities for the mixture are defined by

$$
\begin{array}{r}
n=\sum_{k=1}^{p} n^{k}, \quad \rho=\sum_{k=1}^{p} \rho^{k}, \quad \rho \mathbf{u}=\sum_{k=1}^{p} \rho^{k} \mathbf{u}^{k}, \\
\frac{\rho}{2}\|\mathbf{u}\|^{2}+\mathcal{E}=\sum_{i=1}^{4}\left(\frac{1}{2} \rho^{i}\left\|\mathbf{u}^{i}\right\|^{2}+n^{i} E_{i}\right), \quad \mathcal{E}=\sum_{i=1}^{4} n^{i}\left(\frac{3}{2} k_{B} T+E_{i}\right) .
\end{array}
$$

\section{The Boltzmann equation for reactive gas mixtures}

In this section we recall some backgrounds about the Boltzmann equations for reacting mixtures. In the first subsection we recall the Boltzmann equation for inert gas mixtures which will be considered here. Next in Subsection 3.2 we review some collision operators for reacting mixtures.

3.1. Inert monoatomic gas mixtures. For the sake of simplicity we are going to consider the mechanical Boltzmann equations for inert gas mixtures of $p$ components, that is when collision between molecules are elastic. It reads [3] as

$$
\forall i \in[1, p], \partial_{t} f_{i}+\mathbf{v} \cdot \nabla_{\mathbf{x}} f_{i}=\sum_{k=1}^{k=p} Q_{k i}\left(f_{k}, f_{i}\right):=Q_{i}(\mathbf{f}, \mathbf{f}),
$$

where

$$
Q_{k i}\left(f_{k}, f_{i}\right)=\int_{\mathbb{R}^{3} \times \mathbb{S}^{2}}\left(f_{k}\left(\mathbf{w}_{k i}^{*}\right) f_{i}\left(\mathbf{v}_{k i}^{*}\right)-f_{k}(\mathbf{w}) f_{i}(\mathbf{v})\right) \sigma_{i k}(\boldsymbol{\omega} \cdot \mathbf{V},\|\mathbf{V}\|)\|\mathbf{V}\| d \mathbf{w} d \boldsymbol{\omega} .
$$

Here $Q_{k i}$ is the Boltzmann collision operator between molecules of species $i$ and $k$ and $\sigma_{i k}=\sigma_{k i}$ is the differential cross section which depends on the interaction potential 
between species $i$ and $k$. Finally $\mathbf{V}=\mathbf{w}-\mathbf{v}$ is the relative velocity. The post collisional velocities are given by

$$
\mathbf{v}_{k i}^{*}=\mathbf{v}-2 \frac{m_{k}}{m_{i}+m_{k}}((\mathbf{v}-\mathbf{w}) \cdot \boldsymbol{\omega}) \boldsymbol{\omega}, \quad \mathbf{w}_{k i}^{*}=\mathbf{w}+2 \frac{m_{i}}{m_{i}+m_{k}}((\mathbf{v}-\mathbf{w}) \cdot \boldsymbol{\omega}) \boldsymbol{\omega},
$$

where $m_{i}$ represents the particle mass of species $i$. These equations satisfy the conservation of mass (per species) momentum and energy at microscopic level. The set of collisional invariants $\mathbb{K}$ is spanned by the set of functions $\left(\phi^{l}\right)_{l \in\{1 ; 8\}}$ defined by

$$
\left(\begin{array}{l}
1 \\
0 \\
0 \\
0
\end{array}\right),\left(\begin{array}{l}
0 \\
1 \\
0 \\
0
\end{array}\right),\left(\begin{array}{l}
0 \\
0 \\
1 \\
0
\end{array}\right),\left(\begin{array}{l}
0 \\
0 \\
0 \\
1
\end{array}\right),\left(\begin{array}{c}
m_{1} v_{\alpha} \\
m_{2} v_{\alpha} \\
m_{3} v_{\alpha} \\
m_{4} v_{\alpha}
\end{array}\right),\left(\begin{array}{c}
\frac{1}{2} m_{1} \mathbf{v}^{2}+E_{1} \\
\frac{1}{2} m_{2} \mathbf{v}^{2}+E_{2} \\
\frac{1}{2} m_{3} \mathbf{v}^{2}+E_{3} \\
\frac{1}{2} m_{4} \mathbf{v}^{2}+E_{4}
\end{array}\right),
$$

for $\alpha=x, y, z$.

Collision equilibria are given by Maxwellian distributions

$$
f_{i}=\mathcal{M}_{i}=n_{i}\left(\frac{m_{i}}{2 \pi k_{B} T}\right)^{\frac{3}{2}} \exp \left(-\frac{m_{i}}{2 k_{B} T}(\mathbf{v}-\mathbf{u})^{2}\right),
$$

for any densities $n_{i} \geq 0$, mean velocity $\mathbf{u} \in \mathbb{R}^{3}$, and positive temperature $T . k_{B}$ is the Boltzmann constant.

Next we need to introduce the space $\mathbb{L}^{2}(\mathbf{M})$ equipped with its natural dot product:

$$
\langle\Psi, \Phi\rangle=\sum_{i=1}^{i=p} \int_{\mathbb{R}^{3}} \psi_{i} \phi_{i} \mathcal{M}_{i} d v
$$

The $i^{\text {th }}$ component of the linearized Boltzmann operator reads [3] as

$$
\mathcal{L}_{B_{i}}(\mathbf{g})=\frac{1}{\mathcal{M}_{i}}\left(\sum_{j=1}^{p} Q_{j i}\left(\mathcal{M}_{j}, \mathcal{M}_{i} g_{i}\right)+Q_{j i}\left(\mathcal{M}_{j} g_{j}, \mathcal{M}_{i}\right)\right) .
$$

Then $\operatorname{Ker}\left(\mathcal{L}_{B}\right)=\mathbb{K}, \mathcal{L}_{B}$ is continuous, invertible, and self adjoint negative on $\mathbb{K}^{\perp}$.

3.2. Reacting gas mixtures. In this subsection we recall the three main different approaches leading to collision operators for gas mixtures.

In [32] the authors consider a mixture of four gases undergoing a reversible bimolecular chemical reaction together with mechanical binary collisions. Then the kinetic equation writes for the $i^{\text {th }}$ species as

$$
\frac{\partial f_{i}}{\partial t}+\mathbf{v} \cdot \nabla f_{i}=Q_{i}+J_{i}, \quad i \in\{1 ; 4\}
$$

where $Q_{i}$ is the elastic collision operator and $J_{i}$ represents the chemical collision term. Let us describe briefly $J_{i}$. Let $m_{i}$ (resp. $E_{i}$ ) be the particle mass (resp. the energy of chemical link) for a given species $i$. Then $J_{1}$ is defined by

$$
J_{1}(\mathbf{f})=\int_{\mathbb{R}^{3}} \int_{\mathbb{S}^{2}} U\left(V-\varepsilon_{12}\right) V I_{12}^{34}\left(V, \Omega . \Omega^{\prime}\right)\left(\left(\frac{m_{12}}{m_{34}}\right)^{3} f_{3}\left(\mathbf{v}^{\prime}\right) f_{4}\left(\mathbf{w}^{\prime}\right)-f_{1}(\mathbf{v}) f_{2}(\mathbf{w})\right) d \mathbf{w} d \Omega^{\prime},
$$


where $\left(\mathbf{v}^{\prime}, \mathbf{w}^{\prime}\right)$ are the post-collisional velocities and $m_{i j}=\frac{m_{i} m_{j}}{m_{i}+m_{j}}$ is the reduced mass. The quantity $\varepsilon_{i j}$ is defined by

$$
\varepsilon_{i j}=2 \frac{\Delta E}{m_{i j}} \quad \text { with } \quad \Delta E=E_{3}+E_{4}-E_{1}-E_{2}
$$

$U$ denotes the unit step function and is a threshold for endothermic reactions. This term is linked to the energy of activation which must be given to the system in order that the endothermic reaction can happen.

$I_{12}^{34}\left(V, \Omega . \Omega^{\prime}\right)$ is the cross section where

$$
V=\|\mathbf{w}-\mathbf{v}\|, \Omega=(\mathbf{w}-\mathbf{v}) / V, \quad \Omega^{\prime}=\left(\mathbf{w}^{\prime}-\mathbf{v}^{\prime}\right) /\left\|\mathbf{w}^{\prime}-\mathbf{v}^{\prime}\right\| .
$$

The other chemical terms $J_{i}$ for $i \in\{2 ; 3 ; 4\}$ can be obtained after suitable permutations.

Densities are not conserved but only the total mass. However one must also consider the conservation of atoms number during a reaction. In the case of bimolecular interactions this may be expressed by the following equalities (see [32]):

$$
\int J_{i}(\mathbf{f}) d \mathbf{v}=-\int J_{j}(\mathbf{f}) d \mathbf{v}, \quad(i, j)=(1,3),(1,4),(2,4),
$$

from which one also has

$$
\int J_{1}(\mathbf{f}) d \mathbf{v}=\int J_{2}(\mathbf{f}) d \mathbf{v}, \quad \int J_{3}(\mathbf{f}) d \mathbf{v}=\int J_{4}(\mathbf{f}) d \mathbf{v} .
$$

Finally the conservation of momentum and total energy $\left(\phi^{l}\right)_{l \in\{5 ; 8\}}$ must be added to this set of invariants.

A generalization of this operator for many levels of energy per particles ([25]) can be considered in the framework of Giovangigli and Ern ([20, 23] $)$ and references therein. Each distribution function reads $f_{i}(t, x, v, I)$ where $I$ is the index of the quantum internal energy states of the $i^{t h}$. Then the chemical operator for $f_{1}$ reads

$$
\mathcal{J}_{i}(\mathbf{f})=\sum_{J, K, L} \int\left(f_{3} f_{4} \frac{\beta_{3 K} \beta_{4 L}}{\beta_{1 I} \beta_{2 J}}-f_{i} f_{j}\right) \mathcal{W}_{1234}^{I J K L} d \mathbf{w} d \mathbf{v}^{\prime} d \mathbf{w}^{\prime}
$$

$J, K$, and $L$ are all possible internal quantum energy states of species 2,3 , and 4 . In the case of 25] the ratio $\beta_{3 K} \beta_{4 L} /\left(\beta_{1 I} \beta_{2 J}\right)$ does not depend upon $I, J, K$, and $L$ and simply takes the value $\left(m_{12} / m_{34}\right)^{3}$, while in [23]

$$
\beta_{i I}=\frac{\hbar}{a_{i I} m_{i}^{3}} .
$$

$\hbar$ is the Plank constant and $a_{i I}$ is the degeneracy of the internal energy state $I$ for species $i$. Within our framework we have on one side $a_{i I}=1$ and $\beta_{i I}=\hbar / m_{i}^{3}$. However $\hbar$ does not appear in (3.6) because we are considering bimolecular reaction. For the sake of simplicity we will set $\beta_{i I}=1 / m_{i}^{3}$.

REMARK 3.1. The main difference between (3.6) and (3.9) is the interpretation of the $\mathcal{W}_{1234}^{I J K L}$, which are probabilities of transition [20, 23. However those values as well as the differential cross sections $I_{12}^{34}\left(V, \Omega . \Omega^{\prime}\right)$ are unknown in general. But while angular deviation of the velocities are not accessible from experiments, quite 
accurate evaluations of cross sections (as functions of the translational energy) have been carried out (see e.g. [5]). They allow one to compute the concentrations of the different species at any quantum energy state (vibrational, rotational) as soon as those quantum states can be evaluated for the molecules to be considered. This means that even under strong deviation from equilibrium (except for the translational energies) the chemical processes are quite well evaluated.

REMARK 3.2. One may consider the problem of finding relevant differential cross sections such as to obtain a correct hydrodynamical limit. In this vein one may cite the work of Desvillettes \& co-authors [18, 11] who proposed a model for both mechanical and chemical reactions that allows to recover the energy law of polytropic gases at the Euler limit.

\section{The relaxation model}

4.1. Definition of the model. The distribution function $f_{i}(t, x, v)$ (or for short $f_{i}, i \in[1,4]$ with $\left.\mathbf{f}:=\left(f_{1}, f_{2}, f_{3}, f_{4}\right)\right)$ of a given species $i$ evolves according to the kinetic equation:

$$
\forall i \in[1,4], \partial_{t} f_{i}+\mathbf{v} \cdot \nabla_{\mathbf{x}} f_{i}=\mathcal{R}_{i}^{M E}(\mathbf{f})+\mathcal{R}_{i}^{C E}(\mathbf{f}):=\mathcal{R}_{i}(\mathbf{f}),
$$

where $\mathcal{R}_{i}^{M E}(\mathbf{f})\left(\right.$ resp. $\left.\mathcal{R}_{i}^{C E}(\mathbf{f})\right)$ represents the mechanical (resp. chemical) part of the relaxation operator.

4.1.1. The mechanical BGK operators. The mechanical part of the model is the one constructed in [14. For the sake of clarity we recall how it is derived. Let us now consider the formalism of the thermodynamic of irreversible processes. Mass flux for the $i^{\text {th }}$ species reads

$$
\mathbf{J}_{i}=\sum_{j=1}^{j=4} L_{i j} \nabla\left(\mathcal{G}_{i}^{t r}\right)+L_{i 5} \nabla\left(-\frac{1}{T}\right),
$$

where the specific Gibbs functions $\mathcal{G}_{i}^{\text {tr }}$ are defined by

$$
\mathcal{G}_{i}^{t r}=\frac{k_{B} T}{m_{i}} \ln \left(\frac{n^{i}}{Q_{i}^{t r}}\right), \quad \text { with } \quad Q_{i}^{t r}=\left(2 \pi m_{i} k_{B} T\right)^{\frac{3}{2}}
$$

We may as well consider the full specific Gibbs functions by including partition functions for internal energies. But the present definition is more suited to the derivation of the hydrodynamic limit of our model. We will later on use the full specific Gibbs functions to recast the Navier-Stokes equations in a classical way (Section 5.3.2).

We remark here that $L_{i j}$ and $L_{i 5}$ are of opposite sign of the traditional thermodynamic coefficients. The reason is that they are directly derived from the Boltzmann equation through a Chapman-Enskog expansion. Notice that the density fluxes are generally rather expressed in terms of the phenomenological coefficients, in which case the dependance of the above fluxes on gradients of the densities is called the Fick law. Whatever is the expression the corresponding coefficients depend on temperature $T$ and also on the number densities and molecular masses of each component.

Then in order to recover the matrix $\left(L_{i j}\right)_{i, j=1, \ldots, 4}$ one performs the following steps: 
1. Define the symmetric non positive matrix $L^{*}$ whose elements are

$$
L_{i j}^{*}:=\frac{L_{i j}}{\sqrt{\rho_{i} \rho_{j}} T} .
$$

In the case where the exact Fick coefficients $F_{i j}$ can be measured from experiments one sets

$$
L_{i j}^{*}=\frac{n_{j}}{k_{B} T} F_{i j} \frac{\left(m_{i} m_{j}\right)^{\frac{3}{2}}}{\sqrt{n_{i} n_{j}}} .
$$

It is of course necessary that those coefficients are such that the matrix $L^{*}$ remains symmetric non positive. $L^{*}$ is also related to the diffusion matrix $\left(D_{i j}\right)_{i, j=1, \ldots, 4}$ whose definition is recalled in Section 5.3.2. Then one also has

$$
L_{i j}^{*}=-\frac{\sqrt{\rho_{i} \rho_{j}}}{n k_{B} T} D_{i j}, \quad i, j=1, \ldots, 4 .
$$

This second formula makes possible the computation of $L^{*}$ by using the algorithms developed by Ern and Giovangigli [19].

2. We remark that $L^{*}$ always diagonalizes in an orthonormal basis,

$$
L^{*}=W^{T} K^{*} W
$$

and that up to some permutation in $W$ and $K^{*}$ the corresponding eigenvalues $\left(k_{r}^{*}\right)_{r}$ are non null for $r=1, \ldots, 3$, while $k_{4}^{*}=0$ and corresponds to the conservation of the total mass $\rho$. Then set

$$
\lambda_{r}=-k_{r}^{*-1} \text { for } r=1,2,3 \text { and } \lambda_{4}=0 .
$$

3. Define the vector of velocities $\underline{\mathbf{U}}=\left(\mathbf{u}_{1}, \ldots, \mathbf{u}_{4}\right)^{T}$ with the relation

$$
\underline{\mathbf{U}}-\mathbf{U}=\mathbf{N}^{-1} W^{T}\left(\mathbf{I}-\frac{1}{\nu^{M}} \Delta\right) W \mathbf{N}(\overline{\mathbf{U}}-\mathbf{U}),
$$

where $\mathbf{U}=(\mathbf{u}, \mathbf{u}, \mathbf{u}, \mathbf{u})^{T}, \overline{\mathbf{U}}=\left(\mathbf{u}^{1}, \ldots, \mathbf{u}^{p}\right)^{T}$ and $\mathbf{N}$ and $\Delta$ are the diagonal matrix which diagonal terms are respectively $\left(\sqrt{\rho_{1}}, \ldots, \sqrt{\rho_{4}}\right)$ and $\left(\lambda_{1}, \ldots, \lambda_{4}\right)$.

4. Set

$$
T^{\star}=T-\frac{1}{3 n k_{B}}\left\|W^{T}\left(\mathbf{I}-\frac{1}{\nu^{M}} \Delta\right) W \mathbf{N}(\overline{\mathbf{U}}-\mathbf{U})\right\|^{2} .
$$

DeFinition 4.1. The $i^{\text {th }}$ component of the mechanical operator reads as

$$
\mathcal{R}_{i}^{M E}(\mathbf{f})=\nu^{M}\left(G_{i}-f_{i}\right), i \in[1,4],
$$

with

$$
\forall i \in[1, p], G_{i}=\frac{n^{i}}{\left(2 \pi k_{B} T^{\star} / m_{i}\right)^{3 / 2}} \exp \left(-\frac{m_{i}\left(\boldsymbol{v}-\mathbf{u}_{i}\right)^{2}}{2 k_{B} T^{\star}}\right),
$$

where $\nu^{M}>0$ represents the relaxation coefficient. This coefficient must be chosen with the constraint $\nu^{M} \geq \max _{r} \lambda_{r} / 2$ in order to ensure the positivity of $T^{*}$. 
5. Definition of $\nu^{M}$ : In our case $\nu^{M}$ must be considered as a mean frequency of return to thermodynamic equilibria of the whole set of molecules. Assume that the shear viscosity $\eta$ is given by some formula or approximated by the algorithms of Ern and Giovangigli. Then if $\eta$ satisfies the condition

$$
\eta \leq \frac{n k_{B} T}{\max _{r} \lambda_{r}}
$$

we set

$$
\nu^{M}=n k_{B} T / \eta .
$$

This definition allows us to recover the viscosity $\eta$ at the hydrodynamic limit. It is also classical for the BGK model with one component. However we also have

$$
\lambda_{r}=\frac{n k_{B} T}{d_{r}^{*}}, \quad r=1,2,3, \quad \text { and } \quad \lambda_{4}=0,
$$

where $\left(d_{r}^{*}\right)_{r}$ are the eigenvalues (up to some permutation) of the matrix $\left(\sqrt{\rho_{i} \rho_{j}} D_{i j}\right)_{i j}$ (4.6). Then the condition (4.11) also reads $\eta \leq \min _{r=1,2,3} d_{r}^{*}$ and the definition (4.12) is subjected to the relative speed between the shear stress and diffusion phenomena. For want of anything better we set

$$
\nu^{M}=\max \left(\frac{n k_{B} T}{\eta}, \max _{r} \lambda_{r}\right),
$$

in such a way that the model is always well-posed.

4.1.2. The chemical BGK operators. $\quad \mathcal{R}_{i}^{C E}$ represents the chemical part of the relaxation operator constructed in 24].

Definition 4.2. The $i^{\text {th }}$ component of the chemical operator reads

$$
\mathcal{R}_{i}^{C E}(\mathbf{f})=\nu_{i}^{C}\left(\tilde{\mathcal{M}}_{i}-f_{i}\right),
$$

where $\left(\nu_{i}^{C}\right)_{i}$ are the chemical collision frequencies and

$$
\tilde{\mathcal{M}}_{i}=\tilde{n}^{i}\left(\frac{m_{i}}{2 \pi k_{B} \tilde{T}}\right)^{\frac{3}{2}} \exp \left(-\frac{m_{i}}{2 k_{B} \tilde{T}}(\mathbf{v}-\tilde{\mathbf{u}})^{2}\right) .
$$

It is clear that within the above framework the collision frequency of (e.g.) the first species should read (see [26] or 24])

$$
\frac{1}{n^{1}} \int_{\mathbb{R}^{3}} \int_{\mathbb{S}^{2}} U\left(V-\varepsilon_{12}\right) V I_{12}^{34}\left(V, \Omega . \Omega^{\prime}\right) f_{1}(\mathbf{v}) f_{2}(\mathbf{w}) d \mathbf{w} d \Omega^{\prime},
$$

(if the chemical collision integral is (3.6)). However neither the differential cross section nor the functions $\mathbf{f}$ are known. Recall that the characteristic time of mechanical collisions is supposed to be much smaller than that of the chemical ones. In this case a possible approximation of the chemical terms $\left(J_{i}(\mathbf{f})\right)_{i}$ (3.6) consists in replacing $\mathbf{f}$ with the set of functions at thermodynamical equilibrium M. Further simplificationMaxwellian molecules [26] - allows one to evaluate the collision frequencies which then 
read

$$
\begin{aligned}
& \nu_{1}^{C}=\nu_{12}^{34} \frac{2}{\sqrt{2 \pi}} \Gamma\left(\frac{3}{2}, \frac{\Delta E}{k_{B} T}\right) n^{2}, \\
& \nu_{3}^{C}=\nu_{12}^{34} \frac{2}{\sqrt{2 \pi}} \Gamma\left(\frac{3}{2}, \frac{\Delta E}{k_{B} T}\right)\left(\frac{m_{12}}{m_{34}}\right)^{\frac{3}{2}} \exp \left(\frac{\Delta E}{k_{B} T}\right) n^{4} .
\end{aligned}
$$

Here $\nu_{12}^{34}$ is a physical constant depending on the species involved in the chemical reaction but not on the specified internal energy states $\left(E_{i}\right)_{i=1, \ldots, 4}$ which are considered here. $\Gamma(\alpha, x)$ is the incomplete Gamma function defined by

$$
\Gamma\left(\frac{3}{2}, \frac{\Delta E}{k_{B} T}\right)=\int_{\frac{\Delta E}{k_{B} T}}^{+\infty} \exp ^{-t} t^{\frac{1}{2}} d t=\left(\frac{\Delta E}{k_{B} T}\right)^{\frac{1}{2}} \exp ^{-\frac{\Delta E}{k_{B} T}}+\operatorname{erfc}\left(\sqrt{\frac{\Delta E}{k_{B} T}}\right) .
$$

Thus $\mathcal{N} \Delta E$ is somehow the activation energy of the reaction (1.1) when only one internal energy state per molecule is considered $(\mathcal{N}$ is the Avogadro number).

Notation 4.1. The set of functions $\tilde{\mathbf{M}}=\left(\tilde{\mathcal{M}}_{1}, \tilde{\mathcal{M}}_{2}, \tilde{\mathcal{M}}_{3}, \tilde{\mathcal{M}}_{4}\right)$ is denoted $\tilde{\mathbf{M}}(\boldsymbol{f})$ and respectively $\tilde{\mathcal{M}}_{i}(\mathbf{f})$ for each $i$. We will omit the dependance on $\boldsymbol{f}$ when it is self evident.

We recall how the parameters $\tilde{n}^{i}, \tilde{\mathbf{u}}$, and $\tilde{T}$ are computed. The BGK models must satisfy the same laws as the chemical operators (3.6). That is the conservation of atoms number (3.7) together with the momentum and energy conservation:

$$
\begin{array}{rrrr}
\int\left(\mathcal{R}_{i}^{C E}(\mathbf{f})+\mathcal{R}_{j}^{C E}(\mathbf{f})\right) d \mathbf{v}=0, & (i, j)=(1,3),(1,4),(2,4), \\
\sum_{i=1}^{4} \int m_{i} \mathbf{v} \mathcal{R}_{i}^{C E}(\mathbf{f}) d \mathbf{v}=0, & \sum_{i=1}^{4} \int\left(\frac{1}{2} m_{i} v^{2}+E_{i}\right) \mathcal{R}_{i}^{C E}(\mathbf{f}) d \mathbf{v}=0 .
\end{array}
$$

There are not enough equations to define all unknown densities $\left(\tilde{n}_{i}\right)_{i}$, but it is convenient to write them in the following form:

$$
\tilde{n}_{i}=n^{i}+\Lambda^{i} \frac{\nu_{1}^{C}}{\nu_{i}^{C}}\left(\tilde{n}_{1}-n^{1}\right), \quad i=2,3,4
$$

where $\left(\Lambda^{i}\right)_{i}$ is the string of stoichiometric coefficients $(1,1,-1,-1)$. Thus if $\tilde{n}_{1}$ is known all other densities are defined as well. Then the conservation of the mean velocity together with (4.20) allows one to express $\tilde{\mathbf{u}}$ in term of the macroscopic fields:

$$
\tilde{\mathbf{u}}=\sum_{i=1}^{4} \nu_{i}^{C} m_{i} n^{i} \mathbf{u}^{i} / \sum_{i=1}^{4} \nu_{i}^{C} m_{i} n^{i}
$$

Finally the conservation of the total energy together with (4.20), (4.21) gives the following equation for the temperature $\tilde{T}$ :

$\tilde{T}=\left\{\sum_{i=1}^{4} \nu_{i}^{C} n^{i}\left[\frac{1}{2} m_{i}\left(\left(\mathbf{u}^{i}\right)^{2}-\tilde{\mathbf{u}}^{2}\right)+\frac{3}{2} k_{B} T^{i}\right]+\nu_{1}^{C} \Delta E\left(\tilde{n}_{1}-n^{1}\right)\right\} /\left(\frac{3}{2} k_{B} \sum_{i=1}^{4} \nu_{i}^{C} n^{i}\right)$. 
Thus $\tilde{T}$ is also defined as soon as $\tilde{n}_{1}$ is. This last quantity is obtained by solving the implicit equation

$$
\frac{\nu_{3}^{C} \nu_{4}^{C} \tilde{n}_{1}\left(\nu_{2}^{C} n^{2}+\nu_{1}^{C}\left(\tilde{n}_{1}-n^{1}\right)\right)}{\nu_{2}^{C}\left(\nu_{3}^{C} n^{3}-\nu_{1}^{C}\left(\tilde{n}_{1}-n^{1}\right)\right)\left(\nu_{4}^{C} n^{4}-\nu_{1}^{C}\left(\tilde{n}_{1}-n^{1}\right)\right)} \exp \left(-\frac{\Delta E}{k_{B} \tilde{T}\left(\tilde{n}_{1}\right)}\right)=\left(\frac{m_{12}}{m_{34}}\right)^{\frac{3}{2}} .
$$

As mentioned by the authors of [24, "the left hand side is a monotonically increasing function of $\tilde{n}_{1}$ ranging from 0 to $+\infty$ when $\tilde{n}_{1}$ varies on its domain defined by the constraint of positivity of density and temperature fields. This guarantees existence and uniqueness of the solution to this equation".

4.2. Properties of the model. It is clear that those BGK models share the same collision invariants as the reacting Boltzmann equation itself. Now we show that the model satisfies an entropy dissipation property together with the $\mathrm{H}$ theorem. Let $\mathcal{H}(\mathbf{f})$ be defined by

$$
\mathcal{H}(\mathbf{f})=\sum_{i=1}^{4} \int_{\mathbb{R}^{3}} f_{i} \ln \frac{f_{i}}{m_{i}^{3}} d \mathbf{v} .
$$

Proposition 4.3. The relaxation operators $\mathcal{R}_{i}^{M E}(\mathbf{f})$ and $\mathcal{R}_{i}^{C E}(\mathbf{f})$ satisfy the following dissipative properties:

$$
\sum_{i=1}^{p} \int_{\mathbb{R}^{3}} \mathcal{R}_{i}^{M E}(\mathbf{f}) \ln \left(f_{i} / m_{i}^{3}\right) d \mathbf{v} \leq 0, \quad \sum_{i=1}^{p} \int_{\mathbb{R}^{3}} \mathcal{R}_{i}^{C E}(\mathbf{f}) \ln \left(f_{i} / m_{i}^{3}\right) d \mathbf{v} \leq 0 .
$$

As a consequence $\mathcal{H}$ defined in (4.24) is a Lyapunov function for the whole model.

Proof. On one side side we have $\int \mathcal{R}_{i}^{M E}(\mathbf{f}) d \mathbf{v}=0$ for all $i$ so that

$$
\sum_{i=1}^{4} \int_{\mathbb{R}^{3}} \mathcal{R}_{i}^{M E}(\mathbf{f}) \ln \left(f_{i} / m_{i}^{3}\right) d \mathbf{v}=\sum_{i=1}^{4} \int_{\mathbb{R}^{3}} \mathcal{R}_{i}^{M E}(\mathbf{f}) \ln f_{i} d \mathbf{v} \leq 0
$$

as proved in [14]. On the other side [24] it holds that

$$
\sum_{i=1}^{4} \int_{\mathbb{R}^{3}} \mathcal{R}_{i}^{C E}(\mathbf{f}) \ln \left(f_{i} / m_{i}^{3}\right) d \mathbf{v} \leq 0 .
$$

So Proposition 4.3 follows.

THEOREM 4.4. The following assertions are equivalent:

i) The entropy production rate is equal to 0 , then

$$
\sum_{i=1}^{4} \int_{\mathbb{R}^{3}}\left(\mathcal{R}_{i}^{M E}(\mathbf{f})+\mathcal{R}_{i}^{C E}(\mathbf{f})\right) \ln \left(f_{i} / m_{i}^{3}\right) d \mathbf{v}=0
$$

ii) For all $i$,

$$
\mathcal{R}_{i}^{M E}(\mathbf{f})+\mathcal{R}_{i}^{C E}(\mathbf{f})=0 .
$$

iii) The distribution functions $f_{i}$ are at mechanical and chemical equilibrium:

$$
\forall i \in[1,4], f_{i}=\mathcal{M}_{i}=\frac{n_{i}}{\left(2 \pi k_{B} T / m_{i}\right)^{\frac{3}{2}}} \exp \left(-\frac{m_{i}(\mathbf{v}-\mathbf{u})^{2}}{2 k_{B} T}\right),
$$


with the mass action law

$$
\frac{n^{1} n^{2}}{n^{3} n^{4}}=\left(\frac{m_{12}}{m_{34}}\right)^{3 / 2} \exp \left(\frac{\Delta E}{K T}\right)
$$

Proof.

i) $\Rightarrow$ iii) According to the above Proposition 4.3 i) implies that

$$
\sum_{i=1}^{4} \int_{\mathbb{R}^{3}} \mathcal{R}_{i}^{M E}(\mathbf{f}) \ln \left(f_{i} / m_{i}^{3}\right) d \mathbf{v}=0
$$

This holds [14] if and only if there exists macroscopic values $n_{1}, \ldots, n_{4}, \mathbf{u}, T$ such that

$$
\forall i \in\{1,2,3,4\}, f_{i}=\mathcal{M}_{i}=\frac{n_{i}}{\left(2 \pi k_{B} T / m_{i}\right)^{\frac{3}{2}}} \exp \left(-\frac{m_{i}(\mathbf{v}-\mathbf{u})^{2}}{2 k_{B} T}\right) .
$$

However this condition is necessary but not sufficient for the entropy production rate to vanish. Plugging the above value of the distribution functions into the Definition 4.2 of $\mathcal{R}_{i}^{C E}$, we obtain

$$
\sum_{i=1}^{4} \int_{\mathbb{R}^{3}} \nu_{i}^{C}\left(\tilde{\mathcal{M}}_{i}-\mathcal{M}_{i}\right) \ln \left(\mathcal{M}_{i} / m_{i}^{3}\right) d \mathbf{v}=0
$$

A direct computation (see [24]) together with (4.23) gives

$$
\begin{aligned}
& \sum_{i=1}^{4} \int_{\mathbb{R}^{3}} \nu_{i}^{C}\left(\tilde{\mathcal{M}}_{i}-f_{i}\right) \ln \left(\tilde{\mathcal{M}}_{i} / m_{i}^{3}\right) d \mathbf{v} \\
= & \nu_{1}\left(\tilde{n}_{1}-n_{1}\right)\left[\ln \left(\frac{\tilde{n}_{1} \tilde{n}_{2}}{\tilde{n}_{3} \tilde{n}_{4}}\left(\frac{m_{3} m_{4}}{m_{1} m_{2}}\right)^{\frac{3}{2}}\right)-\frac{\Delta E}{k_{B} \tilde{T}}\right]=0, \quad \forall \mathbf{f} \geq 0 .
\end{aligned}
$$

Subtracting the two previous equations with $f_{i}=\mathcal{M}_{i}$ gives

$$
\sum_{i=1}^{4} \int_{\mathbb{R}^{3}} \nu_{i}^{C}\left(\tilde{\mathcal{M}}_{i}-\mathcal{M}_{i}\right) \ln \frac{\tilde{\mathcal{M}}_{i}}{\mathcal{M}_{i}} d \mathbf{v}=0
$$

The convexity of the function $x \rightarrow x \ln x$ yields $f_{i}=\mathcal{M}_{i}=\tilde{\mathcal{M}}_{i}$ for all $i \in\{1 ; 4\}$ which satisfy the mass action law (4.23).

ii) $\Rightarrow$ i) is trivial.

iii $\Rightarrow$ ii). Assume that $\left(f_{i}\right)_{i}=\left(\mathcal{M}_{i}\right)_{i}$ such that density and temperature fields satisfy the mass action law

$$
\frac{n^{1} n^{2}}{n^{3} n^{4}} \exp \left(-\frac{\Delta E}{k_{B} T}\right)=\left(\frac{m_{12}}{m_{34}}\right)^{3 / 2} .
$$

One has firstly $G_{i}=\mathcal{M}_{i}$ from (4.7) and (4.8) so that $\mathcal{R}_{i}^{M E}(\mathbf{M})=0, \forall i$ (see Definition 4.1). Secondly equations (4.21) and (4.22) give $\tilde{\mathbf{u}}=\mathbf{u}$ and

$$
\tilde{T}=T+\nu_{1}^{C} \Delta E\left(\tilde{n}_{1}-n^{1}\right) /\left(\frac{3}{2} k_{B} \sum_{i=1}^{4} \nu_{i}^{C} n^{i}\right) .
$$

One obtains the same equation as (4.31) by setting $\tilde{n}_{1}=n_{1}$ into (4.23). Thus $n_{1}$ is the unique solution of (4.23) and $\tilde{n}_{i}=n_{i}, \forall i$ according to (4.20). One also has $\tilde{T}=T$ from (4.32) so that $\tilde{\mathcal{M}}_{i}=\mathcal{M}_{i}, \forall i$ and $\mathcal{R}_{i}^{C E}(\mathbf{f})=0$. 


\section{Hydrodynamic limit for slow reactions}

The slow reactions regime corresponds to the situation where the chemistry characteristic time is much smaller than the mechanical ones. This regime has been studied in (19]) by estimating the ratio between the characteristic time of mechanical collisions and the chemistry characteristic time. This ratio is shown to be less than 0.16 for temperatures varying between 300 and $1000 \mathrm{~K}$. The hydrodynamic limit of our model is then obtained by studying the equations obtained from

$$
\forall i \in[1,4], \partial_{t} f_{i}^{\varepsilon}+\mathbf{v} \cdot \nabla_{x} f_{i}^{\varepsilon}=\frac{1}{\varepsilon} \mathcal{R}_{i}^{M E}(\mathbf{f})+\mathcal{R}_{i}^{C E}(\mathbf{f})
$$

as $\varepsilon \rightarrow 0$.

5.1. Asymptotic expansion. The classical Chapman-Enskog expansion consists in setting

$$
\forall i \in[1,4], f_{i}^{\varepsilon}=f_{i}^{0}+\varepsilon f_{i}^{1}+\varepsilon f_{i}^{2} \ldots
$$

and plugging those expressions in (5.1). Then we get, at order -1 in $\varepsilon, \mathcal{R}_{i}^{M}(\mathbf{f})=0$ for all $i$. So $f_{i}^{0}=\mathcal{M}_{i}$ (see [14).

Setting $f_{i}^{1}=\mathcal{M}_{i} g_{i}$ the equation (5.1) at order 0 read

$$
\partial_{t} \mathcal{M}_{i}+\mathbf{v} \cdot \nabla_{x} \mathcal{M}_{i}=\mathcal{M}_{i} \mathcal{L}_{i}^{M E}(\mathbf{g})+\mathcal{R}_{i}^{C E}(\mathbf{M}), \quad \forall i,
$$

where $\mathcal{L}_{i}^{M E}$ is the $i^{t h}$ component of the linearized operator of $\mathcal{R}^{M E}$ around the Maxwellian distributions $\mathbf{M}$. Its $i^{t h}$ component is defined as usual by the formula

$$
D \mathcal{R}_{i}^{M E}(\mathbf{M}) . \mathbf{M g}=\mathcal{M}_{i} \mathcal{L}_{i}^{M E}(\mathbf{g})=\lim _{\tau \rightarrow 0} \frac{\mathcal{R}_{i}^{M E}(\mathbf{M}(1+\tau \mathbf{g}))-\mathcal{R}_{i}^{M E}(\mathbf{M})}{\tau},
$$

where $D \mathcal{R}^{M E}(\mathbf{M}) . \mathbf{M g}$ represents the differential of $\mathcal{R}^{M E}$ at $\mathbf{M}$ in the direction $\mathbf{M g}$. After computing $\partial_{t} \mathcal{M}_{i}$ from the Euler system (5.9, 5.10, 5.11) in function of the space derivatives, the equation (5.3) will in turn be particularly easy to solve thanks to the exact formulation of the (pseudo-)inverse of $\mathcal{L}^{M E}$.

In order to obtain an asymptotic expansion of (5.1) up to order 1 in $\varepsilon$, the term $\mathcal{R}_{i}^{M E}(\mathbf{f})$ must be expanded up to order 2. Setting $f_{i}^{2}=\mathcal{M}_{i} h_{i}$, then the equation (5.1) up to the order 1 is derived from

$$
\begin{aligned}
& \partial_{t}\left(\mathcal{M}_{i}\left(1+\varepsilon g_{i}\right)\right)+\mathbf{v} \cdot \nabla_{x}\left(\mathcal{M}_{i}\left(1+\varepsilon g_{i}\right)\right) \\
= & \frac{1}{\varepsilon} \mathcal{R}_{i}^{M E}\left(\mathbf{M}\left(1+\varepsilon \mathbf{g}+\varepsilon^{2} \mathbf{h}\right)\right)+\mathcal{R}_{i}^{C E}(\mathbf{M}(1+\varepsilon \mathbf{g})) .
\end{aligned}
$$

The Taylor expansion of $\mathcal{R}_{i}^{M E}\left(\mathbf{M}\left(1+\varepsilon \mathbf{g}+\varepsilon^{2} \mathbf{h}\right)\right)$ gives

$$
\begin{aligned}
& \mathcal{R}_{i}^{M E}\left(\mathbf{M}\left(1+\varepsilon \mathbf{g}+\varepsilon^{2} \mathbf{h}\right)\right) \\
= & \mathcal{R}_{i}^{M E}(\mathbf{M})+\varepsilon \mathcal{L}_{i}^{M E}(\mathbf{g})+\varepsilon^{2}\left(\mathcal{L}_{i}^{M E}(\mathbf{h})+\frac{1}{2} D^{2} \mathcal{R}_{i}^{M E}(\mathbf{M}) \cdot(\mathbf{M g}, \mathbf{M g})\right)+\mathcal{O}\left(\varepsilon^{3}\right) .
\end{aligned}
$$

Next one must expand $\mathcal{R}_{i}^{C E}(\mathbf{M}(1+\varepsilon \mathbf{g}))$ as well. The linearized operator $\mathcal{L}^{C E}(\mathbf{g})$ around $\mathbf{M}$ is defined with

$$
\begin{aligned}
D \mathcal{R}_{i}^{C E}(\mathbf{M}) \cdot \mathbf{M g}=\mathcal{L}_{i}^{C E}(\mathbf{g}) & =\lim _{\tau \rightarrow 0} \frac{1}{\tau}\left(\mathcal{R}_{i}^{C E}(\mathbf{M}(1+\tau \mathbf{g}))-\mathcal{R}_{i}^{C E}(\mathbf{M})\right) \\
& =\lim _{\tau \rightarrow 0} \frac{1}{\tau}\left(\tilde{\mathcal{M}}_{i}\left(\mathbf{M}(1+\tau \mathbf{g})-\tilde{\mathcal{M}}_{i}(\mathcal{M})-\tau g_{i} \mathcal{M}_{i}\right) .\right.
\end{aligned}
$$


We remark that we have not factorized $\mathcal{M}_{i}$ in front of $\mathcal{L}_{i}^{C E}$ in this definition. The reason is that such a factor should not appear, as suggested by the last line of the above expression. Hence the expansion up to order 1 in $\varepsilon$ of (5.5) gives

$$
\begin{aligned}
& \partial_{t}\left(\mathcal{M}_{i}\left(1+\varepsilon g_{i}\right)\right)+\mathbf{v} \cdot \nabla_{x}\left(\mathcal{M}_{i}\left(1+\varepsilon g_{i}\right)\right) \\
= & \mathcal{L}_{i}^{M E}(\mathbf{g})+\mathcal{R}_{i}^{C E}(\mathbf{M})+\varepsilon\left(\mathcal{L}_{i}^{C E}(\mathbf{g})+\mathcal{L}_{i}^{M E}(\mathbf{h})+\frac{1}{2} D^{2} \mathcal{R}_{i}^{M E}(\mathbf{M}) \cdot(\mathbf{M g}, \mathbf{M g})\right)+\mathcal{O}\left(\varepsilon^{2}\right)
\end{aligned}
$$

REMARK 5.1. According to their definition, the second order terms in $\varepsilon$ of (5.6) conserve mass, total momentum, and energy. Therefore they will neither contribute to the Euler system nor to the Navier-Stokes system.

5.2. The Euler system. The Euler equations are classically obtained by integration of the 8 first moments of (5.3). They read

$$
\begin{aligned}
\partial_{t} \rho^{i}+\nabla_{x} \cdot\left(\rho^{i} \mathbf{u}\right) & =m_{i} \Lambda_{i} \nu_{1}^{C}\left(\tilde{n}_{1}-n^{1}\right), \\
\partial_{t}(\rho \mathbf{u})+\nabla_{x} \cdot(\rho \mathbf{u} \otimes \mathbf{u}+p \mathbb{I}) & =0 \\
\partial_{t} E_{t o t}+\nabla \cdot\left(E_{t o t} \mathbf{u}+\mathbb{P} \mathbf{u}\right) & =0
\end{aligned}
$$

where the total energy and the pressure are

$$
E_{\text {tot }}=\frac{1}{2} \rho \mathbf{u}^{2}+\mathcal{E}, \quad \mathbb{P}=n k_{B} T \mathbb{I},
$$

and $\mathbb{I}$ is the identity matrix.

\subsection{The Navier-Stokes system.}

5.3.1. Setting of the problem. In order to obtain the Navier-Stokes system we have to perform the following steps:

1. Compute $\mathbf{g}$ solution to the equation (5.3) by using the Euler system.

2. Compute the linearized operator $\mathcal{L}^{C E}$ by using formula (5.7).

3. Integrate (5.8) with respect to the elements of $\mathbb{K}(3.1)$ and keep all terms up to the order 1 in $\varepsilon$.

Those steps are performed in the following sections and the final result is given in Proposition 5.1

Proposition 5.1. The Navier-Stokes system for slow chemical reactions of (5.1) reads

$$
\begin{aligned}
& \partial_{t} \rho^{i}+\nabla \cdot\left(\rho^{i} \mathbf{u}\right)+\varepsilon \nabla \cdot \boldsymbol{J}_{i}=m_{i} \Lambda_{i} \nu_{1}^{C}\left(\tilde{n}_{1}-n_{1}\right)+\varepsilon \omega_{i}^{1}, \\
& \partial_{t}(\rho \mathbf{u})+\nabla \cdot(\rho \mathbf{u} \otimes \mathbf{u}+\mathbb{P})+\varepsilon \nabla \cdot\left(\mathbb{J}_{\mathbf{u}}\right)=0, \\
& \quad \partial_{t} E_{t o t}+\nabla \cdot\left(E_{t o t} \mathbf{u}+\mathbb{P} \mathbf{u}\right)+\varepsilon \nabla \cdot\left(\mathbb{J}_{\mathbf{u}}[\mathbf{u}]\right)+\varepsilon \nabla \cdot \boldsymbol{J}_{\boldsymbol{q}}=0,
\end{aligned}
$$

where the fluxes are defined by

$$
\boldsymbol{J}_{i}=\sum_{j=1}^{j=4} L_{i j} \nabla\left(\frac{\mathcal{G}_{i}^{t r}}{T}\right), \quad \mathbb{J}_{\mathbf{u}}=-\frac{n k_{B} T}{\nu^{M}} \mathbb{D}(\boldsymbol{u}), \quad \boldsymbol{J}_{q}=-\kappa \nabla T+\sum_{i} E_{i} \boldsymbol{J}_{i},
$$

and the partial thermal conductivity for monoatomic gases is

$$
\kappa=\frac{5 k_{B}^{2} T}{2 \nu^{M}} \sum_{i=1}^{4} \frac{n^{i}}{m_{i}}
$$


The matrix $L_{i j}$ is defined in (4.2) and the Specific Gibbs functions $\left(\mathcal{G}_{i}^{t r}\right)_{i}$ in (4.3). $\mathbb{D}(\mathbf{u})$ is the traceless part of the deformation tensor

$$
\mathbb{D}(\mathbf{u})=\frac{1}{2}\left[\nabla \mathbf{u}+(\nabla \mathbf{u})^{T}\right]-\frac{1}{3}(\nabla \cdot \mathbf{u}) \mathbb{I} .
$$

$\left(m_{i} \Lambda_{i} \nu_{1}^{C}\left(\tilde{n}_{1}-n_{1}\right)\right)_{i}$ are the zero order chemical terms where $\tilde{n}_{1}$ is the unique solution to the equation (4.23). Finally the first order perturbation of the chemical term reads

$$
\begin{gathered}
\omega_{i}^{1}=m_{i} \Lambda_{i} \nu_{1}^{C} \tau\left(\tilde{n}_{1}\right) \bar{h}(\mathbf{g}) \\
\tau\left(\tilde{n}_{1}\right)=\left(\frac{m_{12}}{m_{34}}\right)^{\frac{3}{2}} \frac{\Delta E}{k_{B} \tilde{T}^{2}} /\left(\xi^{\prime}\left(\tilde{n}_{1}\right)+\xi\left(\tilde{n}_{1}\right) \frac{\nu_{1}(\Delta E)^{2}}{\frac{3}{2} k_{B}^{2} \tilde{T}^{2} \sum_{i=1}^{4} \nu_{i}^{C} n^{i}}\right), \\
\bar{h}(\mathbf{g})=\frac{1}{\nu^{M}} \Delta E\left(\tilde{n}_{1}-n^{1}\right) \frac{\sum_{i=1}^{4}\left(\nu_{i}^{C}\right)^{2} \rho_{i}}{\sum_{i=1}^{4} \nu_{i}^{C} n^{i}}\left(\frac{\nu_{1}^{C}}{\sum \nu_{i}^{C} n^{i}}-\frac{1}{n}\right) .
\end{gathered}
$$

REMARK 5.2. When all chemical relaxation frequencies are equal the first order chemical perturbation $\omega_{i}^{1}$ vanishes. Moreover for fixed $(t, \mathbf{x})$ the densities $\left(\tilde{n}_{i}\right)_{i}$ are solutions to the local mass action law

$$
\frac{\tilde{n}_{1} \tilde{n}_{2}}{\tilde{n}_{3} \tilde{n}_{4}} \exp \left(-\frac{\Delta E}{k_{B} \tilde{T}\left(\tilde{n}_{1}\right)}\right)=\left(\frac{m_{12}}{m_{34}}\right)^{\frac{3}{2}}
$$

together with the relations

$$
\begin{aligned}
\tilde{n}_{i}-n^{i} & =\Lambda^{i}\left(\tilde{n}_{1}-n^{1}\right), \quad i=2,3,4, \\
\tilde{T} & =T+\Delta E\left(\tilde{n}_{1}-n^{1}\right) /\left(\frac{3}{2} k_{B} n\right) .
\end{aligned}
$$

In a thermodynamic framework-which considers the space homogeneous problem related to (5.12) and (5.14) with initial conditions $n^{i}(0)=n^{i}, \forall i$ and $T(0)=T$ - those solutions correspond to the "true" chemical equilibrium states (when $t \rightarrow+\infty$ ) of the chemical reaction (1.1).

Remark 5.3. According to the expression of the fluxes given in Proposition 5.1, the shear viscosity $\eta$ and partial thermal conductivity for monoatomic species $\kappa$ are equal to

$$
\eta=\frac{n k_{B} T}{\nu^{M}}, \quad \kappa=\frac{5 k_{B}^{2} T}{2 \nu^{M}} \sum_{i=1}^{4} \frac{n^{i}}{m_{i}} .
$$

Define the mean molar mass of the mixture $\bar{m}$ as

$$
\bar{m}=\frac{\mathcal{N} \rho}{\sum_{i=1}^{4} \frac{\rho^{i}}{m_{i}}}=\frac{\mathcal{N} \rho}{n} .
$$

Then the Prandtl number $\operatorname{Pr}$ in the monoatomic setting can be computed as

$$
\operatorname{Pr}=\frac{5}{2} \frac{R}{\bar{m}} \frac{\eta}{\kappa}=\frac{n^{2}}{\rho \sum_{i=1}^{4} \frac{n^{i}}{m_{i}}}
$$

where $R=\mathcal{N} k_{B}$ is the constant of perfect gases. Hence in the situation of indifferentiability - when all molecules are the same - the Prandtl number is equal to 1 , which is a drawback of the classical BGK model for a single monoatomic species. 
5.3.2. Other formulation of the Navier-Stokes equations. It is clear that the present model does not pretend to reproduce all phenomena that occur for polyatomic gas mixtures. However it is possible to recast the above equations in a more familiar and useful framework such as that of the book of Giovangigli 23. We first introduce the specific enthalpy and specific Gibbs free energy for each species. They are defined by

$$
h_{i}=\frac{5}{2} \frac{k_{B} T}{m_{i}}+\frac{E_{i}}{m_{i}}, \quad \mathcal{G}_{i}=\frac{k_{B} T}{m_{i}} \ln \left(\frac{n^{i}}{Q_{i}}\right), \quad Q_{i}=\left(2 \pi m_{i} k_{B} T\right)^{\frac{3}{2}} \exp \left(-\frac{E_{i}}{k_{B} T}\right),
$$

where we have introduced the partition function for internal energy

$$
Q_{i}^{i n t}=\exp \left(-E_{i} / k_{B} T\right)
$$

of the species $i$. Those definitions are similar to those introduced in [23] when the molecules have multiple internal energy states. From this the mass fluxes can be conveniently rewritten in the form

$$
\mathbf{J}_{i}=\sum_{j=1}^{4} \tilde{L}_{i j} \nabla\left(\frac{\mathcal{G}_{j}}{T}\right)+\tilde{L}_{i 5} \nabla\left(-\frac{1}{T}\right)
$$

where $\mathbf{J}_{i}$ is defined in (4.2) and

$$
\tilde{L}_{i j}=L_{i j}, \quad i, j=1, \ldots, 4, \quad \tilde{L}_{i 5}=\sum_{j=1}^{4} L_{i j} \frac{E_{j}}{m_{j}}, \quad i=1, \ldots, 4 .
$$

Then the heat flux $\mathbf{J}_{\mathbf{q}}$ (5.15) may be written in a "symmetric" form

$$
\mathbf{J}_{\mathbf{q}}=\sum_{i=1}^{4} \tilde{L}_{5 i} \nabla\left(\frac{\mathcal{G}_{j}}{T}\right)+\tilde{L}_{55} \nabla\left(-\frac{1}{T}\right),
$$

where $\tilde{L}_{5 i}=\tilde{L}_{i 5}$ and

$$
\tilde{L}_{55}=\sum_{i, j=1}^{4} \frac{E_{i}}{m_{i}} L_{i j} \frac{E_{j}}{m_{j}}-\kappa T^{2}<0
$$

( $\kappa$ is given in (5.16) ). The null space of $\tilde{L}$ is $\mathbb{R}(1,1,1,1,0)^{T}$ and $\tilde{L}$ is symmetric non positive as shows the decomposition $\tilde{L}=P^{t} \bar{L} P+M$ with

$$
M=\left[\begin{array}{lllll}
0 & 0 & 0 & 0 & 0 \\
0 & 0 & 0 & 0 & 0 \\
0 & 0 & 0 & 0 & 0 \\
0 & 0 & 0 & 0 & 0 \\
0 & 0 & 0 & 0 & \tilde{L}_{55}
\end{array}\right], \quad P=\left[\begin{array}{lllll}
1 & 0 & 0 & 0 & \frac{E_{1}}{m_{1}} \\
0 & 1 & 0 & 0 & \frac{E_{1}}{m_{2}} \\
0 & 0 & 1 & 0 & \frac{E_{3}}{m_{3}} \\
0 & 0 & 0 & 1 & \frac{E_{4}}{m_{4}} \\
0 & 0 & 0 & 0 & 1
\end{array}\right], \quad \bar{L}=\left[\begin{array}{ccccc}
\tilde{L}_{11} & \tilde{L}_{12} & \tilde{L}_{13} & \tilde{L}_{14} & 0 \\
\tilde{L}_{21} & \tilde{L}_{22} & \tilde{L}_{23} & \tilde{L}_{24} & 0 \\
\tilde{L}_{31} & \tilde{L}_{32} & \tilde{L}_{33} & \tilde{L}_{34} & 0 \\
\tilde{L}_{41} & \tilde{L}_{42} & \tilde{L}_{43} & \tilde{L}_{44} & 0 \\
0 & 0 & 0 & 0 & 1
\end{array}\right] .
$$

We are now going to use the work of Kurochkin et al. [30] that bridges the TIP formulations of the fluxes with more classical and practical ones. This comparison is still possible in our case because the thermodynamic functions are clearly defined. 
Denote with $A \in \mathbb{R}^{5 \times 5}$ the matrix

$$
A=\left[\begin{array}{ccccc}
1 & 0 & 0 & 0 & -h_{1} \\
0 & 1 & 0 & 0 & -h_{2} \\
0 & 0 & 1 & 0 & -h_{3} \\
0 & 0 & 0 & 1 & -h_{4} \\
0 & 0 & 0 & 0 & 1
\end{array}\right] .
$$

Next we define $\hat{L}=A^{T} \tilde{L} A$. Then it is easily checked that the mass and heat fluxes read

$$
\begin{aligned}
J_{i} & =-\sum_{j=1}^{p} \rho^{i} D_{i j} \frac{\nabla p_{j}}{p}-\rho^{i} \theta_{i} \frac{\nabla T}{T}, \quad \forall i \in\{1 ; 4\}, \\
\mathbf{J}_{\mathbf{q}} & =-\hat{\lambda} \nabla T-p \sum_{i=1}^{4} \theta_{i} \frac{\nabla p_{i}}{p}+\sum_{i=1}^{4} h_{i} J_{i} .
\end{aligned}
$$

Here $\left(p_{i}=n^{i} k_{B} T\right)_{i}$ are the partial pressures and the diffusion matrix $\left(D_{i j}\right)_{i, j=1, \ldots, 4}$, thermal diffusion coefficients $\left(\theta_{i}\right)_{i=1, \ldots, 4}$, and partial thermal conductivity $\hat{\lambda}$ are respectively found to be

$$
D_{i j}=-\frac{n k_{B} L_{i j}}{\rho_{i} \rho_{j}}, \quad \theta_{i}=\frac{\hat{L}_{i 5}}{\rho_{i} T}, \quad \hat{\lambda}=\frac{\hat{L}_{55}}{T^{2}} .
$$

Hence we recover the structural form of the fluxes in terms of phenomenological coefficients. We remark that the relation between the diffusion matrix and $\left(L_{i j}\right)_{i, j=1, \ldots, 4}$ allows us to approximate the latter by using the algorithm developed by Ern and Giovangigli ([19]). As a consequence the mechanical model $R^{M E}$ (Section 4.1.1) is made explicit once the shear viscosity $\eta$ is known. This is also given by the above approximation.

Nevertheless the thermal diffusion coefficients and partial thermal conductivity are incorrect as can be seen from the formulae (5.20) and (5.22). Indeed the values of $\left(\tilde{L}_{i 5}\right)_{i}$ and $\left(\tilde{L}_{55}\right)_{i}$ are not the "real" values coming from the thermodynamics of irreversible processes because our mechanical model just allows us to recover the matrix $\left(L_{i j}\right)_{i, j=1, \ldots, 4}$ at the hydrodynamic limit.

Let us now compare $\mathbb{J}_{\mathbf{u}}$ in (5.15) with the general form that holds for a mixture of polyatomic gases with chemical reactions, which writes as

$$
\mathbb{J}_{\mathbf{u}}=p^{r e a c} \mathbb{I}-\alpha \nabla \cdot \mathbf{u} \mathbb{I}-\eta \mathbb{D}(\mathbf{u}),
$$

where $p^{r e a c}$ is the chemical pressure, $\alpha$ is the volume viscosity, and $\eta$ is the shear viscosity. In our case, because $\alpha=0$ we are only considering elastic collisions. But it is possible to recover the exact shear viscosity under suitable conditions (see Section 4.1.1). Finally there is no chemical pressure in our case. This is due to the (extremely) limited number of possible quantum energy states per molecules.

Finally the zero-order molecular production rate of the $i^{\text {th }}$ species (5.12) is quite different from the Maxwellian production rate of the chemical operators (3.6). That is,

$$
\nu_{i}^{C}\left(\tilde{n}_{i}-n_{i}\right) \neq \Lambda_{i} \int_{\mathbb{R}^{3}} \mathcal{J}_{1}(\mathbf{M}) d \mathbf{v}
$$

Only the negative parts can be compared if the frequencies are chosen such as in (5.2). 
5.3.3. Computation of $\mathrm{g}$. Before we set the first result we need to introduce some notations.

Definition 5.2. $\boldsymbol{C}_{i}$ is the vector whose $i^{\text {th }}$ component is $\mathbf{v}-\mathbf{u}$ and others are $0 . \mathcal{P}_{\mathbb{K}}$ is the orthogonal projection on $\mathbb{K}$ with respect to the scalar product (3.3) and $\mathcal{I}$ is the identity operator.

Lemma 5.3. The perturbation $\mathbf{g}$ reads

$$
\mathbf{g}=\left(\mathcal{L}^{M E}\right)^{-1}\left(\Psi^{M E}\right)-\frac{1}{\nu^{M}} \Psi^{C E}=\mathbf{g}^{M E}+\mathbf{g}^{C E},
$$

where

$$
\begin{gathered}
\Psi_{i}^{M E}=\left(\sum_{j=1}^{j=p} k_{B}^{-1}\left(\mathcal{I}-\mathcal{P}_{\mathbb{K}}\right) \boldsymbol{C}_{j} \cdot \nabla\left(\frac{\mu_{j}}{k_{B} T}\right)+\mathbb{A}: \mathbb{D}(\mathbf{u})+\boldsymbol{B} \cdot \nabla\left(\frac{1}{k_{B} T}\right)\right)_{i} \\
\Psi_{i}^{C E}=-\nu_{i}^{C} \frac{\tilde{\mathcal{M}}_{i}-\mathcal{M}_{i}}{\mathcal{M}_{i}}+\nu_{i}^{C} \frac{\tilde{n}_{i}-n_{i}}{n_{i}} \\
+\frac{2}{3 n k_{B}^{2} T^{2}}\left(\frac{1}{2} m_{i}(\mathbf{v}-\mathbf{u})^{2}-\frac{3}{2} k_{B} T\right) \nu_{1}^{C}\left(\tilde{n}_{1}-n_{1}\right) \Delta E
\end{gathered}
$$

$\mathbb{A}$ and $\boldsymbol{B}$ are the list of tensors defined by

$$
(\mathbb{A})_{i}=\frac{m_{i}}{k_{B} T}\left[(\mathbf{v}-\mathbf{u}) \otimes(\mathbf{v}-\mathbf{u})-\frac{1}{3}(\mathbf{v}-\mathbf{u})^{2} \mathbb{I}\right],(\boldsymbol{B})_{i}=(\mathbf{v}-\mathbf{u})\left[\frac{5}{2} k_{B} T-\frac{1}{2} m_{i}(\mathbf{v}-\mathbf{u})^{2}\right] .
$$

$\mathcal{I}$ is the identity and $\mathcal{P}_{\mathbb{K}}$ is the operator of projection on $\mathbb{K}$ with respect to the scalar product (3.3). Finally $\boldsymbol{C}_{i}$ is the vector in $\mathbb{R}^{3 \times 4}$ whose $i^{\text {th }}$ line is $\boldsymbol{v}-\boldsymbol{u}$ and the others are 0 .

Proof. Using the Euler equations (5.9, 5.10, 5.11) the time derivatives of the macroscopic fields in (5.3) can be expressed in terms of spatial derivatives. Then we use the formalism of [14] which can be easily extended to the case of slow chemical reactions. This reads

$$
\begin{aligned}
\mathcal{L}^{M E}(\mathbf{g})= & \sum_{j=1}^{j=p} k_{B}^{-1}\left(\mathcal{I}-\mathcal{P}_{\mathbb{K}}\right) m_{i}\left(\mathbf{C}_{j}\right) \cdot \nabla\left(\mathcal{G}_{j}^{t r}\right)+\mathbb{A}: \mathbb{D}(\mathbf{u})+\mathbf{B} \cdot \nabla\left(\frac{1}{k_{B} T}\right) \\
& -\left(\mathcal{I}-\mathcal{P}_{\mathbb{K}}\right) \mathcal{R}^{C E}(\mathbf{M}) .
\end{aligned}
$$

Let us first give more details of

$$
\mathcal{R}_{i}^{C E}(\mathbf{M})=\nu_{i}^{C}\left(\tilde{\mathcal{M}}_{i}-\mathcal{M}_{i}\right),
$$

which corresponds to the chemical contribution. $\tilde{n}_{i}$, $\tilde{\mathbf{u}}$, and $\tilde{T}$ are defined by the relations (4.20, 4.21, 4.22) corresponding to the fields of $\tilde{\mathbf{M}}$. Hence we have

$$
\begin{array}{r}
\tilde{n}_{i}=n_{i}+\Lambda^{i} \frac{\nu_{1}^{C}}{\nu_{i}^{C}}\left(\tilde{n}_{1}-n^{1}\right), \quad i=2,3,4, \\
\tilde{\mathbf{u}}=\mathbf{u}, \quad \tilde{T}=T+\nu_{1}^{C} \Delta E\left(\tilde{n}_{1}-n^{1}\right) /\left(\frac{3}{2} k_{B} \sum_{i=1}^{4} \nu_{i}^{C} n_{i}\right),
\end{array}
$$


where $\tilde{n}_{1}$ is the unique solution of the implicit equation (4.23). It is then an easy task to compute the $i^{\text {th }}$ line of $\left(\mathcal{I}-\mathcal{P}_{\mathbb{K}}\right) \mathcal{R}^{C E}(\mathbf{M})$ by using the definition of $\mathbb{K}^{\perp}$ and the result reads

$$
\begin{aligned}
& \Psi_{i}^{C E} \\
= & -\nu_{i}^{C} \frac{\tilde{\mathcal{M}}_{i}-\mathcal{M}_{i}}{\mathcal{M}_{i}}+\nu_{i}^{C} \frac{\tilde{n}_{i}-n_{i}}{n_{i}}+\frac{2}{3 n k_{B}^{2} T^{2}}\left(\frac{1}{2} m_{i}(\mathbf{v}-\mathbf{u})^{2}-\frac{3}{2} k_{B} T\right) \times \nu_{1}^{C}\left(\tilde{n}_{1}-n_{1}\right) \Delta E .
\end{aligned}
$$

We now need to compute $\mathcal{L}^{M E^{-1}}\left(\Psi_{i}^{M E}+\Psi_{i}^{C E}\right)$. Recall that

$$
\forall \phi \in \mathbb{K}^{\perp}, \quad\left(\mathcal{L}^{M E}\right)^{-1}(\phi)=\frac{1}{\nu^{M}}\left(\left(R-\mathcal{I}_{\mathbb{C}}\right)^{-1} \circ \mathcal{P}_{\mathbb{C}}+\left(\mathcal{P}_{\mathbb{C}}-\mathcal{I}_{\mathbb{C}}\right)\right)(\phi),
$$

(Lemma 8 in [14]) where $\mathbb{C}$ is the space spanned by the vectors

$$
\left(\mathcal{I}-\mathcal{P}_{\mathbb{K}}\right)\left(\mathbf{C}_{i}\right), \quad i=1, \ldots, 4,
$$

and $R$ is a linear operator acting on $\mathbb{C}$. Then one has $\mathbf{g}^{M E}=\left(\mathcal{L}^{M E}\right)^{-1}\left(\Psi_{i}^{M E}\right)$, from which one can compute the "elastic" part of the fluxes (see [14]). Next recall that

$$
\left(\mathcal{I}-\mathcal{P}_{\mathbb{K}}\right) \mathbf{C}_{i}=\sum_{j} a_{i j} \mathbf{C}_{i}, \quad\left(a_{i j}\right)_{i, j} \in \mathbb{R}^{4} \times \mathbb{R}^{4},
$$

and one can easily check that $\Psi^{C E} \in \mathbb{C}^{\perp}$. So Lemma 5.3 follows.

REMARK 5.4. The decomposition of the perturbation $\mathbf{g}$ into a mechanical and a chemical part corresponds to the usual decomposition that is valid for the reactive Boltzmann equation given in 23.

5.4. Computation of $\mathcal{L}^{C E}$. In order to compute $\mathcal{L}^{C E}(\mathrm{~g})$ from formula (5.7) we need a preliminary result.

Lemma 5.4. Let $\tilde{\mathcal{M}}_{i}^{g}$ be the "attractors" of the chemical term $\mathcal{R}_{i}^{C E}(\mathbf{M}(1+\varepsilon \mathbf{g}))$. Then

$$
\tilde{\mathcal{M}}_{i}^{g}(\mathbf{v})=\tilde{n}_{i}^{g}\left(\frac{m_{i}}{2 \pi k_{B} \tilde{T}_{g}}\right)^{\frac{3}{2}} \exp \left(-m_{i} \frac{\left(\mathbf{v}-\tilde{\mathbf{u}}_{g}\right)^{2}}{2 k_{B} \tilde{T}_{g}}\right),
$$

with

$$
\begin{gathered}
\tilde{n}_{i}^{g}=\tilde{n}_{i}+\varepsilon \Lambda_{i} \frac{\nu_{1}^{C}}{\nu_{i}^{C}} \tau\left(\tilde{n}_{1}\right) \bar{h}(\mathbf{g})+\mathcal{O}\left(\varepsilon^{2}\right), \\
\tilde{\mathbf{u}}_{g}=\mathbf{u}+\varepsilon \frac{\sum_{i=1}^{4} \nu_{i}^{C} m_{i} n_{i} u_{i}^{g}}{\sum_{i=1}^{4} \nu_{i}^{C} m_{i} n_{i}}, n_{i} u_{i}^{g}=\int_{\mathbb{R}^{3}} \mathcal{M}_{i} g_{i} \mathbf{v} d \mathbf{v}, \mathbf{u}^{g}=\left(u_{1}^{g}, u_{2}^{g}, u_{3}^{g}, u_{4}^{g}\right), \\
\tilde{T}_{g}=\tilde{T}+\varepsilon \bar{h}(\mathbf{g})\left(\frac{\nu_{1}^{C} \Delta E \tau\left(\tilde{n}_{1}\right)}{\frac{3}{2} k_{B} \sum_{i=1}^{4} \nu_{i}^{C} n_{i}}+1\right)+\mathcal{O}\left(\varepsilon^{2}\right),
\end{gathered}
$$

where

$$
\tilde{T}=T+\nu_{1}^{C} \Delta E\left(\tilde{n}_{1}-n^{1}\right) /\left(\frac{3}{2} k_{B} \sum_{i=1}^{4} \nu_{i}^{C} n^{i}\right),
$$


and

$$
\bar{h}(\mathbf{g})=\frac{1}{\nu^{M}} \Delta E\left(\tilde{n}_{1}-n_{1}\right) \frac{\sum_{i=1}^{4}\left(\nu_{i}^{C}\right)^{2} \rho_{i}}{\sum_{i=1}^{4} \nu_{i}^{C} n_{i}}\left(\frac{\nu_{1}^{C}}{\sum \nu_{i}^{C} n_{i}}-\frac{1}{n}\right) .
$$

This tedious and lengthy proof is left to appendix. Then expanding $\tilde{\mathcal{M}}_{i}^{g}$ around $\varepsilon$ and using formula (5.7) we obtain

$$
\begin{aligned}
\mathcal{L}_{i}^{C E}(\mathbf{g})= & \nu_{i}^{C} \widetilde{\mathcal{M}}_{i}\left(\Lambda_{i} \frac{\nu_{1}^{C}}{\nu_{i}^{C}} \frac{\tau\left(\tilde{n}_{1}\right)}{\tilde{n}_{i}} \bar{h}(\mathbf{g})-\frac{\bar{h}(\mathbf{g})}{2 \pi k_{B} \tilde{T}}+\frac{m_{i}}{2 \pi k_{B} \tilde{T}}\left(2\left\langle\mathbf{v}-\mathbf{u}, \mathbf{u}^{g}\right\rangle\right.\right. \\
& \left.\left.+\frac{(\mathbf{v}-\mathbf{u})^{2}}{2 k_{B} \tilde{T}} \bar{h}(\mathbf{g})\right)\right)-\nu_{i}^{C} \mathcal{M}_{i} g_{i} .
\end{aligned}
$$

REMARK 5.5. $\bar{h}(\mathbf{g})=0$ (5.18) when the collision frequencies $\left(\nu_{i}^{C}\right)$ are equal. Hence in this case there are no contributions of order $\varepsilon$ to the chemical term in the NavierStokes equations for the evolution of densities.

Remark 5.6. The expression (5.38) of $\mathcal{L}_{i}^{C E}$ (g) does not depend on the mechanical perturbation $\mathrm{g}^{M E}$ (5.26). Then in contrast to [23] there are no derivatives of the macroscopic fields in the first order production chemical terms.

\subsection{Computation of the right-hand side of Navier Stokes system.} This subsection is devoted to the end of the proof of Proposition 5.1.

Proof. (Proposition 5.1). In order to obtain a Navier-Stokes system we have to consider for any $i \in\{1,2,3\}$ the moments of $\mathcal{M}_{i}\left(1+\varepsilon g_{i}\right)$. Writing $g_{i}=g_{i}^{M E}+g_{i}^{C E}$ we remark that fluxes of order $\varepsilon$ depend as expected only on $g_{i}^{M E}$. Hence we can directly infer that the left hand sides of the Navier-Stokes equations are those obtained in (14]). Denote that one important point is the computation of matrix $\left(L_{i j}\right)$ appearing in the definition of the mass flux $J_{i}$. More precisely, using (5.32) we obtain $L_{i j}$ through the expression of the Onsager matrix from the Chapmann-Enskog expansion.

Next one has to compute the chemical terms up to order $\varepsilon$. As concerns the equations for densities those terms read

$$
\int_{\mathbb{R}^{3}}\left(\mathcal{R}_{i}^{C E}(\mathbf{M})+\varepsilon \mathcal{L}_{i}^{C E}(\mathbf{g})\right) d \mathbf{v}=\Lambda_{i} \nu_{1}^{C}\left(\tilde{n}_{1}-n_{1}\right)+\varepsilon \Lambda_{i} \nu_{1}^{C} \tau\left(\tilde{n}_{1}\right) \bar{h}(\mathbf{g}) .
$$

Recall that

$$
\mathcal{R}_{i}^{C E}(\mathbf{M}(1+\varepsilon \mathbf{g}))=\mathcal{R}_{i}^{C E}(\mathbf{M})+\varepsilon \mathcal{L}_{i}^{C E}(\mathbf{g})+\mathcal{O}\left(\varepsilon^{2}\right)
$$

and that $\mathcal{R}^{C E}(\mathbf{f})$ satisfies equation (4.19) for any function $\mathbf{f}=\left(f_{1}, f_{2}, f_{3}, f_{4}\right)$. Then we have on one side

$$
\sum_{i} m_{i} \int_{\mathbb{R}^{3}}\left(\mathcal{R}_{i}^{C E}(\mathbf{M})+\varepsilon \mathcal{L}_{i}^{C E}(\mathbf{g})\right) \mathbf{v} d \mathbf{v}=\mathcal{O}\left(\varepsilon^{2}\right),
$$

and on the other side

$$
\sum_{i} \int_{\mathbb{R}^{3}}\left(\frac{1}{2} m_{i} \mathbf{v}^{2}+E_{i}\right)\left(\mathcal{R}_{i}^{C E}(\mathbf{M})+\varepsilon \mathcal{L}_{i}^{C E}(\mathbf{g})\right) d \mathbf{v}=\mathcal{O}\left(\varepsilon^{2}\right),
$$

which finishes the proof. 


\section{Discussion and conclusion}

The BGK model which is proposed here features the same structure as the simplified Boltzmann equation for a reacting gas mixture proposed by Rossani and Spiga [32] or of the more general Boltzmann equation with different levels of internal energies 23. That is, a sum of two different terms for the nonreactive and the reactive "collisions". Let us recall that our model is designed here to satisfy good mathematical properties $(\mathrm{H}$-theorem, ...) and to present a splitting method that seems naturally adapted to the case of slow chemical reactions. The Chapman-Enskog expansion clearly outlines the respective contributions of the first order mechanical and chemical perturbations (5.26). As expected all transport coefficients (thermal diffusion, viscosity, Fourier law, ...) depend only on the modeling of the mechanical part of the BGK model. Its construction is based on the knowledge of the Fick law (4.5) or of the diffusion matrix (4.6) together with the shearn viscosity. In some cases the Fick law may be obtained from experiments but the corresponding matrix of coefficients must be symmetrical non positive. This property is satisfied if the diffusion matrix is approximated by algorithms developed by Ern and Giovangigli [19]. The hydrodynamic limit of the model features good structural agreement (Section 5.3.2) with the usual Navier-Stokes for polyatomic gases 23. (Section [5.3.2). The correct diffusion matrix is recovered by construction. This holds also for the shear viscosity under the suitable condition (4.11). But the thermal diffusion coefficients and partial thermal conductivity are incorrect. There is no volume viscosity as expected because we are only dealing with elastic collisions and molecules with only one fixed internal energy state. As a consequence there is also no chemical pressure.

The zero order chemistry source terms (5.12) are not equal to the Maxwellian production rates of the real chemical operators, in contrast to what is obtained from the theoretical BGK model of Bisi and Spiga [9] and for the Grad approximation in [8]. But positivity of temperature of the attractors and the H-theorem are guaranteed at the kinetic level unlike the model in $[9$. Remark that perturbations of order $\varepsilon$ are free from any derivatives of the spatial gradients as in 8 . One should also point out that those perturbations vanish when the chemical frequencies are equal.

Finally a generalization of the mechanical operator as well as a construction of a chemical operator allowing one to recover the Maxwellian production rates in the general case of polyatomic gases are in progress.

Appendix A. Proof of Lemma 5.4.

Proof. As $g \in \mathbb{K}^{\perp}$,

$$
\int_{\mathbb{R}^{3}} \mathcal{M}_{i}\left(1+\varepsilon g_{i}\right) d \mathbf{v}=n^{i}
$$

We first want to establish a transcendental equation as (24]) for which $\tilde{n}_{1}^{g}$ is a solution. By using the mass conservation for $i \in\{2 ; 3 ; 4\}$,

$$
\tilde{n}_{i}^{g}=n^{i}+\Lambda_{i} \frac{\nu_{1}^{C}}{\nu_{i}^{C}}\left(\tilde{n}_{1}^{g}-n^{1}\right)
$$

Next the conservation of the impulsion for the chemical operator gives

$$
\sum_{i=1}^{4} m_{i} \nu_{i}^{C} \int_{\mathbb{R}^{3}}\left(\tilde{\mathcal{M}}_{i}^{g}-\mathcal{M}_{i}\left(1+\varepsilon g_{i}\right)\right) \mathbf{v} d \mathbf{v}=0 .
$$


So defining $u_{i}^{g}$ by

$$
n^{i} u_{i}^{g}=\int_{\mathbb{R}^{3}} \mathcal{M}_{i} g_{i} \mathbf{v} d \mathbf{v}
$$

it holds that

$$
\tilde{\mathbf{u}}_{g}=\mathbf{u}+\varepsilon \frac{\sum_{i=1}^{4} \nu_{i}^{C} m_{i} n^{i} u_{i}^{g}}{\sum_{i=1}^{4} \nu_{i}^{C} m_{i} n^{i}}=\mathbf{u}+\varepsilon f\left(u_{g}\right)
$$

where

$$
f\left(u_{g}\right)=\frac{\sum_{i=1}^{4} \nu_{i}^{C} m_{i} n^{i} u_{i}^{g}}{\sum_{i=1}^{4} \nu_{i}^{C} m_{i} n^{i}} .
$$

Hence by setting

$$
T_{i}^{g}=\frac{m_{i}}{3 k_{B} n^{i}} \int_{\mathbb{R}^{3}}\left(\mathbf{v}-\mathbf{u}-\varepsilon \mathbf{u}_{i}^{g}\right)^{2} \mathcal{M}_{i}\left(1+\varepsilon g_{i}\right) d \mathbf{v}
$$

we finally obtain the following expression for $\tilde{T}_{g}$ :

$$
\tilde{T}_{g}=\frac{\left(\sum_{i=1}^{4} \frac{1}{2} \nu_{i}^{C} m_{i} n^{i}\left(\left(u+\varepsilon u_{i}^{g}\right)^{2}-\left(u+\varepsilon f\left(u_{g}\right)\right)^{2}\right)+\sum_{i=1}^{4} \nu_{i}^{C} \frac{3}{2} k_{B} T_{i}^{g}+\nu_{1}^{C} \Delta E\left(\tilde{n}_{1}^{g}-n^{1}\right)\right)}{\frac{3}{2} k_{B} \sum_{i=1}^{4} \nu_{i}^{C} n^{i}} .
$$

In order to simplify (A.4) we note that

$$
\begin{aligned}
& \sum_{i=1}^{4} \frac{1}{2} \nu_{i}^{C} m_{i} n^{i}\left(\left(u+\varepsilon u_{i}^{g}\right)^{2}-\left(u+\varepsilon f\left(u_{g}\right)\right)^{2}\right) \\
= & \sum_{i=1}^{4} \nu_{i}^{C} m_{i} n^{i}\left(2 \varepsilon u \cdot u_{i}^{g}-2 \varepsilon u \cdot f\left(u_{g}\right)+\varepsilon^{2} u_{i}^{g 2}-\varepsilon^{2} f\left(u_{g}\right)^{2}\right) \\
= & 2 \varepsilon u \cdot\left(\sum_{i=1}^{4} \nu_{i}^{C} m_{i} n^{i} u_{i}^{g}-f\left(u_{g}\right) \sum_{i=1}^{4} \nu_{i}^{C} m_{i} n^{i}\right)+\varepsilon^{2} \sum_{i=1}^{4} \nu_{i}^{C} m_{i} n^{i}\left(u_{i}^{g}-f\left(u_{g}\right)^{2}\right) \\
= & \varepsilon^{2} \sum_{i=1}^{4} \nu_{i}^{C} m_{i} n^{i}\left(u_{i}^{g 2}-f\left(u_{g}\right)^{2}\right)
\end{aligned}
$$

according to the definition of $f\left(u_{g}\right)$ A.2. The relation (A.3) leads to

$$
\begin{gathered}
T_{i}^{g}=\frac{m_{i}}{3 k_{B} n^{i}}\left(\int_{\mathbb{R}^{3}}(\mathbf{v}-\mathbf{u})^{2} \mathcal{M}_{i}\left(1+\varepsilon g_{i}\right) d \mathbf{v}-2 \varepsilon u_{i}^{g} \cdot \int_{\mathbb{R}^{3}}(\mathbf{v}-\mathbf{u}) \mathcal{M}_{i}\left(1+\varepsilon g_{i}\right) d \mathbf{v}\right. \\
\left.+\varepsilon^{2}\left(u_{i}^{g}\right)^{2} \int_{\mathbb{R}^{3}} \mathcal{M}_{i}\left(1+\varepsilon g_{i}\right) d \mathbf{v}\right) .
\end{gathered}
$$


Hence by setting

$$
h_{i}^{g}=\frac{m_{i}}{3 k_{B} n^{i}} \int_{\mathbb{R}^{3}}(\mathbf{v}-\mathbf{u})^{2} \mathcal{M}_{i} g_{i} d \mathbf{v},
$$

we see that

$$
T_{i}^{g}=T+\varepsilon h_{i}^{g}-\frac{\varepsilon^{2}}{2 k_{B}}\left(u_{i}^{g}\right)^{2} .
$$

So

$$
\frac{3}{2} k_{B} \sum_{i=1}^{4} \nu_{i}^{C} n^{i} T_{i}^{g}=\frac{3}{2} k_{B} T\left(\sum_{i=1}^{4} \nu_{i}^{C} n^{i}+\varepsilon \sum_{i=1}^{4} \nu_{i}^{C} n^{i} h_{i}^{g}-\frac{\varepsilon^{2}}{3 k_{B}} \sum_{i=1}^{4} \nu_{i}^{C} n^{i}\left(u_{i}^{g}\right)^{2}\right) .
$$

Finally, A.4 leads to

$$
\tilde{T}_{g}=T+\nu_{1}^{C}\left(\frac{\Delta E\left(\tilde{n}_{1}^{g}-n^{1}\right)}{\frac{3}{2} k_{B} \sum_{i=1}^{4} \nu_{i}^{C} n^{i}}\right)+\varepsilon \bar{h}(\mathbf{g})+\varepsilon^{2} \bar{l}(\mathbf{g}),
$$

with

$$
\begin{aligned}
\bar{h}(\mathbf{g}) & =\frac{\sum_{i=1}^{4} \nu_{i}^{C} n^{i} h_{i}^{g}}{\sum_{i=1}^{4} \nu_{i}^{C} n^{i}}, \\
\bar{l}(\mathbf{g}) & =\frac{\sum_{i=1}^{4} \nu_{i}^{C} n^{i} m_{i}\left(\left(u_{i}^{g}\right)^{2}-\left(f\left(u_{g}\right)\right)^{2}\right)-\frac{1}{2} \sum_{i=1}^{4} \nu_{i}^{C} n^{i} m_{i} u_{i}^{g}}{\sum_{i=1}^{4} \nu_{i}^{C} n^{i}} .
\end{aligned}
$$

Next we aim to compute $\tilde{n}_{1}^{g}$. Let us write the implicit equation (4.23) in the following way:

$$
F\left(\varepsilon, \tilde{n}_{1}^{g}\right)=\xi\left(\tilde{n}_{1}^{g}\right) \exp \left(-\frac{\Delta E}{k_{B}\left(\tilde{T}_{M}\left(\tilde{n}_{1}^{g}\right)+\varepsilon \bar{h}(\mathbf{g})+\varepsilon^{2} \bar{l}(\mathbf{g})\right)}\right)-\left(\frac{m_{12}}{m_{34}}\right)^{\frac{3}{2}}=0,
$$

where

$$
\xi\left(\tilde{n}_{1}^{g}\right)=\frac{\nu_{3}^{C} \nu_{4}^{C} \tilde{n}_{1}^{g}\left(\nu_{2}^{C} n^{2}+\nu_{1}^{C}\left(\tilde{n}_{1}^{g}-n^{1}\right)\right)}{\nu_{2}^{C}\left(\nu_{3}^{C} n^{3}+\nu_{1}^{C}\left(\tilde{n}_{1}^{g}-n^{1}\right)\right)\left(\nu_{4}^{C} n^{4}+\nu_{1}^{C}\left(\tilde{n}_{1}^{g}-n^{1}\right)\right)}
$$

and

$$
\tilde{T}_{M}\left(\tilde{n}_{1}^{g}\right)=T+\nu_{1}^{C} \frac{\Delta E\left(\tilde{n}_{1}^{g}-n^{1}\right)}{\frac{3}{2} k_{B} \sum_{i=1}^{4} \nu_{i}^{C} n^{i}} .
$$

The computation of $\tilde{n}_{1}^{g}$ is performed through the implicit function theorem at $\varepsilon=0$. If $\varepsilon=0, \tilde{n}_{1}^{g}=\tilde{n}_{1}$ and

$$
F\left(0, \tilde{n}_{1}\right)=\xi\left(\tilde{n}_{1}\right) \exp \left(-\frac{\Delta E}{\tilde{T} k_{B}}\right)-\left(\frac{m_{12}}{m_{34}}\right)^{\frac{3}{2}}=0 .
$$

So from (A.8) $\xi\left(\tilde{n}_{1}\right)$ reads

$$
\xi\left(\tilde{n}_{1}\right)=\left(\frac{m_{34}}{m_{12}}\right)^{\frac{3}{2}} \exp \left(\frac{\Delta E}{\tilde{T} k_{B}}\right)
$$


Then by using the implicit function theorem $\tilde{n}_{1}^{g}$ can be expanded in $\varepsilon$ :

$$
\tilde{n}_{1}^{g}=\tilde{n}_{1}+\varepsilon \frac{\frac{\partial}{\partial x} F\left(0, \tilde{n}_{1}\right)}{\frac{\partial}{\partial y} F\left(0, \tilde{n}_{1}\right)}+\mathcal{O}\left(\varepsilon^{2}\right) .
$$

From (A.7) it comes that

$$
\begin{aligned}
\frac{\partial}{\partial x} F\left(0, \tilde{n}_{1}\right) & =\xi\left(\tilde{n}_{1}\right) \frac{\Delta E \bar{h}(\mathbf{g})}{k_{B} \tilde{T}} \exp \left(-\frac{\Delta E}{k_{B} \tilde{T}}\right) \\
& =\left(\frac{m_{12}}{m_{34}}\right)^{\frac{3}{2}} \frac{\Delta E \bar{h}(\mathbf{g})}{k_{B} \tilde{T}^{2}} \exp \left(-\frac{\Delta E}{k_{B} \tilde{T}}\right)
\end{aligned}
$$

and

$$
\frac{\partial}{\partial y} F\left(0, \tilde{n}_{1}\right)=\left(\xi^{\prime}\left(\tilde{n}_{1}\right)+\xi\left(\tilde{n}_{1}\right) \frac{\nu_{1}(\Delta E)^{2}}{\frac{3}{2} k_{B}^{2} \tilde{T}^{2} \sum_{i=1}^{4} \nu_{i}^{C} n^{i}}\right) \exp \left(-\frac{\Delta E}{k_{B} \tilde{T}}\right) .
$$

Next by introducing $\tau\left(\tilde{n}_{1}\right)$ defined in (5.17), $\tilde{n}_{1}^{g}$ writes as $\tilde{n}_{1}^{g}=\tilde{n}_{1}+\varepsilon \tau\left(\tilde{n}_{1}\right) \bar{h}(\mathbf{g})+\mathcal{O}\left(\varepsilon^{2}\right)$. Hence according to (A.1) $\tilde{n}_{i}^{g}$ satisfies the relation (5.30) for all $i \in\{2 ; 3 ; 4\}$. Moreover, by plugging the expression of $\tilde{n}_{1}^{g}$ into (A.6), it holds that $\tilde{T}_{g}$ can be defined from relation (5.36).

\section{Computation of $\bar{h}(\mathbf{g})$.}

Firstly as

$$
\begin{aligned}
\int_{\mathbb{R}^{3}}(\mathbf{v}-\mathbf{u})(\mathbf{v}-\mathbf{u})^{2} \mathcal{M}_{i} d \mathbf{v}=0, & \int_{\mathbb{R}^{3}} \mathbb{A}_{i}(\mathbf{v}-\mathbf{u})(\mathbf{v}-\mathbf{u})^{2} \mathcal{M}_{i} d \mathbf{v}=0, \\
& \int_{\mathbb{R}^{3}} B_{i}(\mathbf{v}-\mathbf{u})(\mathbf{v}-\mathbf{u})^{2} \mathcal{M}_{i} d \mathbf{v}=0,
\end{aligned}
$$

the mechanical part $g_{i}^{M E}$ of $g_{i}$ can be removed in (A.5) and $h_{i}^{g}$ reduces to

$$
h_{i}^{g}=\frac{m_{i}}{2 k_{B} n^{i}} \int_{\mathbb{R}^{3}} \mathcal{M}_{i}(\mathbf{v}-\mathbf{u})^{2} g_{i}^{C E} d \mathbf{v} .
$$

That is,

$$
\begin{aligned}
h_{i}^{g}= & \frac{m_{i}}{2 \nu^{M} k_{B} n^{i}} \int_{\mathbb{R}^{3}}(\mathbf{v}-\mathbf{u})^{2}\left(\nu_{i}^{C}\left(\widetilde{\mathcal{M}}_{i}-\mathcal{M}_{i}\right)-\nu_{i}^{C} \mathcal{M}_{i}\left(\tilde{n}_{i}-n^{i}\right)\right. \\
& \left.\quad-\frac{\nu_{1}^{C E} \Delta E}{3 n k_{B}^{2} T^{2}}\left(\tilde{n}_{1}-n^{1}\right)\left(\frac{1}{2} m_{i}(\mathbf{v}-\mathbf{u})^{2}-\frac{3}{2} k_{B} T\right)\right) \mathcal{M}_{i} d \mathbf{v} \\
= & \frac{3 \nu_{i}^{C} m_{i}}{2 \nu^{M} n^{i}} \tilde{n}_{i}\left(\tilde{T}_{M}\left(\tilde{n}_{1}\right)-T\right)-\frac{\nu_{i}^{C}}{\nu^{M} n k_{B}} \Delta E\left(\tilde{n}_{1}-n^{1}\right) \\
= & \frac{\nu_{i}^{C}}{\nu^{M} k_{B}} \Delta E\left(\tilde{n}_{1}-n^{1}\right)\left(\frac{\nu_{1}^{C}}{\sum \nu_{i}^{C} n^{i}}-\frac{1}{n}\right),
\end{aligned}
$$

where we have used (5.37) in the last equation. Finally we obtain

$$
\bar{h}(\mathbf{g})=\Delta E\left(\tilde{n}_{1}-n^{1}\right) \frac{\sum_{i=1}^{4}\left(\nu_{i}^{C}\right)^{2} \rho_{i}}{\nu^{M} \sum_{i=1}^{4} \nu_{i}^{C} n^{i}}\left(\frac{\nu_{1}^{C}}{\sum \nu_{i}^{C} n^{i}}-\frac{1}{n}\right) .
$$




\section{REFERENCES}

[1] P. Andries, K. Aoki, and B. Perthame, A consistent BGK-type model for gas mixtures, J. Stat. Phys., 106(5/6), 993-1018, 2002.

[2] P. Andries, P. LeTallec, J.P. Perlat, and B. Perthame, Entropy condition for the ES BGK model of Boltzmann equation for mono and polyatomic gases, Eur. J. Mech. (B fluids) 19, 813-830, 2000 .

[3] K. Aoki, C. Bardos, and S. Takata, Knudsen layer for a gas mixture, J. Stat. Phys., 112(3/4), 629-655, 2003.

[4] P.L. Bhatnagar, E.P. Gross, and M. Krook, A model for collision processes in gases, Phy. Rev., 94, 511-524, 1954.

[5] F.F.W. McCourt, J.J. Beenaker, W.E. Khler, and I. Kuscer, Nonequilibrium Phenomena in Polyatomic Gases, Oxford University Press, 2, 1990.

[6] M. Bisi and L. Desvilettes, From reactive Boltzmann equations to reaction-diffusion systems, J. Stat. Phys., 124, 2-4, 881-912, 2006.

[7] M. Bisi and L. Desvilettes, Some remarks about the scaling of systems of reactive Boltzmann equations, Kin. Rel. Mod., 1(4), 515-520, 2008.

[8] M. Bisi, M. Groppi, and G. Spiga, Grad's closure in the kinetic theory of a chemically reacting gas, Cont. Mech. Thermodyn., 14(2), 207-222, 2002.

[9] M. Bisi and G. Spiga, On a kinetic BGK model for slow chemical reactions, Kin. Rel. Mod., 4(1), 163-167, 2011.

[10] M. Bisi, M. Groppi, and G. Spiga, Kinetic Approach to Chemically Reacting Gas Mixtures, Modelling and Numerics of Kinetic Dissipative Systems, Nova Sci. Publ., Hauppauge, NY, 2006.

[11] J.F. Bourgat, L. Desvillettes, P. Le Tallec, and B. Perthame, Microreversible collisions for polyatomic gases and Boltzmann's theorem, Eur. J. Mech., (B-Fluids), 13(2), 237-254, 1994.

[12] S. Brull and J. Schneider, A new approach of the Ellipsoidal statistical model, Cont. Mech. Thermodyn., 20(2), 63-74, 2008.

[13] S. Brull and J. Schneider, On the ellipsoidal statistical model for polyatomic gases, Cont. Mech. Thermodyn., 20(8), 489-508, 2009.

[14] S. Brull, V. Pavan, and J. Schneider, Derivation of BGK models for mixtures, Eur. J. Mech. (B-Fluids), 33, 74-86, 2012.

[15] G. Chaban, R. Jaffe, D.W. Schwenke, and W. Huo, Dissociation Cross Sections and Rate Coefficients for Nitrogen from Accurate Theoretical Calculations, 46th AIAA Aerospace Science Meeting and Exhibit, AIAA 2008.

[16] S. Chapman and T.G. Cowling, The Mathematical Theory of Non Uniform Gases, Third Edition, Cambridge University Press, Cambridge, 1970.

[17] L. Desvilettes, Sur un modèle de type Borgnakke-Larsen conduisant à des lois d'énergies non linéaires en température pour les gaz parfaits polyatomiques, Ann. Fac. Sci. Toulouse, 257262, 1997.

[18] L. Desvillettes, R. Monaco, and F. Salvarani, A kinetic model allowing to obtain the energy law of polytropic gases in the presence of chemical reactions, Eur. J. Mech. (B-Fluids), 24, 219-236, 2005.

[19] A. Ern and V. Giovangigli, Multicomponent Transport Algorithms, Lecture Notes in Physics, New Series Monographs, 1994.

[20] A. Ern and V. Giovangigli, The kinetic chemical equilibrium regime, Physica A, 260, 49-72, 1998.

[21] S.R. de Groot and P. Mazur, Nonequilibrium Thermodynamics, North-Holland, Amsterdam, 1962.

[22] V. Garzo, A. Santos, and J.J. Brey, A kinetic model for a multicomponent gas, Phys. of Fluid A1 N2, 380-383, 1989.

[23] V. Giovangigli, Multicomponent flow modeling, Science China Mathematics, 55(2), 285-308, 2012.

[24] M. Groppi, S. Rjasanow, and G. Spiga, A kinetic relaxation approach to fast reactive mixtures: Shock wave structure, J. Stat. Mech. Theory. Exp., 10, P10010, 2009.

[25] M. Groppi and G. Spiga, Kinetic approach to chemical reactions and inelastic transitions in a rarefied gas, J. Math. chem., 26, 197-219, 1999.

[26] M. Groppi and G. Spiga, A Bhatnagar-Gross-Krook approach for chemically reacting mixtures, Phys. Fluids, 16(12), 4273-4284, 2004.

[27] P.L. Gross and M. Krook, Model for collision processes in gases: Small-amplitude oscillations of charged two-component systems, Phys. Rev., 102 N3, 593-604, 1956.

[28] L.H. Holway, New statistical models for kinetic theory: Methods of construction, Phys. Fluids 
9, 1958-1673, 1966 .

[29] S. Kosuge, Model Boltzmann equation for gas mixtures: Construction and numerical comparison, Eur. J. Mech. (B-Fluids), 170-184, 2009.

[30] V.I. Kurochkin, S.F. Makarenko, and G.A. Tirskii, Transport coefficients and the Onsager relations in the kinetic theroy of dense gas mixtures, J. Appl. Mech. Tech. Phys., 25, 218$225,1984$.

[31] D. Levermore, Moment closure hierarchies for kinetic theories, J. Stat. Phys., 83(5-6), 1021$1065,1996$.

[32] A. Rossani and G. Spiga, A note on the kinetic theory of chemically reacting gases, Physica A, $272,563-573,1999$

[33] J. Schneider, Entropic approximation in kinetic theory, M2AN, 38(3), 541-561, 2004. 